\title{
HIGH-RESOLUTION RADIO OBSERVATIONS OF THE REMNANT OF SN 1987A AT HIGH FREQUENCIES
}

\author{
Giovanna Zanardo ${ }^{1}$, L. Staveley-Smith ${ }^{1,2}$, C.-Y. NG ${ }^{3}$, B. M. Gaensler ${ }^{2,4}$, \\ T. M. PotTer ${ }^{1}$, R. N. MANChester ${ }^{5}$, AND A. K. TZIoumis ${ }^{5}$ \\ ${ }^{1}$ International Centre for Radio Astronomy Research (ICRAR), M468, The University of Western Australia, \\ Crawley, WA 6009, Australia; giovanna.zanardo@icrar.org \\ ${ }^{2}$ Australian Research Council, Centre of Excellence for All-sky Astrophysics (CAASTRO) \\ ${ }^{3}$ Department of Physics, The University of Hong Kong, Pokfulam Road, Hong Kong \\ ${ }^{4}$ Sydney Institute for Astronomy (SIfA), School of Physics, The University of Sydney, NSW 2006, Australia \\ ${ }^{5}$ CSIRO Astronomy and Space Science, Australia Telescope National Facility, P.O. Box 76, Epping, NSW 1710, Australia \\ Received 2012 November 15; accepted 2013 January 26; published 2013 April 1
}

\begin{abstract}
We present new imaging observations of the remnant of Supernova (SN) 1987A at 44 GHz, performed in 2011 with the Australia Telescope Compact Array (ATCA). The 0!'35 × 0!'23 resolution of the diffraction-limited image is the highest achieved to date in high-dynamic range. We also present a new ATCA image at $18 \mathrm{GHz}$ derived from 2011 observations, which is super-resolved to $0^{\prime} \cdot 25$. The flux density is $40 \pm 2 \mathrm{mJy}$ at $44 \mathrm{GHz}$ and $81 \pm$ $6 \mathrm{mJy}$ at $18 \mathrm{GHz}$. At both frequencies, the remnant exhibits a ring-like emission with two prominent lobes, and an east-west brightness asymmetry that peaks on the eastern lobe. A central feature of fainter emission appears at $44 \mathrm{GHz}$. A comparison with previous ATCA observations at 18 and $36 \mathrm{GHz}$ highlights higher expansion velocities of the remnant's eastern side. The $18-44 \mathrm{GHz}$ spectral index is $\alpha=-0.80\left(S_{v} \propto v^{\alpha}\right)$. The spectral index map suggests slightly steeper values at the brightest sites on the eastern lobe, whereas flatter values are associated with the inner regions. The remnant morphology at $44 \mathrm{GHz}$ generally matches the structure seen with contemporaneous $\mathrm{X}$-ray and $\mathrm{H} \alpha$ observations. Unlike the $\mathrm{H} \alpha$ emission, both the radio and $\mathrm{X}$-ray emission peaks on the eastern lobe. The regions of flatter spectral index align and partially overlap with the optically visible ejecta. Simple free-free absorption models suggest that emission from a pulsar wind nebula or a compact source inside the remnant may now be detectable at high frequencies or at low frequencies if there are holes in the ionized component of the ejecta.
\end{abstract}

Key words: acceleration of particles - circumstellar matter - ISM: supernova remnants - radiation mechanisms: non-thermal - radio continuum: general - supernovae: individual (SN 1987A)

Online-only material: color figures

\section{INTRODUCTION}

Radio supernovae ( $\mathrm{SNe}$ ) result from the collision between a supernova shock and the progenitor's circumstellar medium (CSM). As the interaction between the propagating blast wave and the CSM drives the particle acceleration process, supernova remnants (SNRs) are natural laboratories for studying particle spectra and their variation in time (see Weiler et al. 2002, for a review).

Supernova 1987A in the Large Magellanic Cloud, as the only nearby core-collapse supernova observed to date, has provided a unique opportunity to study the evolution of the $\mathrm{SN}-\mathrm{CSM}$ interaction in young radio remnants. The complex CSM distribution in the SNR is believed to have originated from a red supergiant (RSG) which evolved into a blue supergiant (BSG) about 20,000 years before the explosion (Crotts \& Heathcote 2000). Models of the progenitor evolution suggest that the equatorially denser, i.e., slower, RSG wind (Blondin \& Lundqvist 1993; Martin \& Arnett 1995) was swept up by the faster BSG wind (Morris \& Podsiadlowski 2007), thus forming high density rings. In particular, beside the central circular ring in the equatorial plane (equatorial ring, ER), observations with the Hubble Space Telescope (HST) have also revealed two outer rings that formed from the mass loss of the progenitor star, located on either side of the equatorial plane (Jakobsen et al. 1991; Plait et al. 1995), which confer to SNR 1987A a peculiar triple-ring nebula structure.

Since the radio detection of the remnant in mid-1990 (Turtle et al. 1990), the synchrotron emission has been generated by the shock wave propagating into the ring-shaped distribution of the CSM in the equatorial plane. Monitoring of the flux density has been regularly undertaken with the Molonglo Observatory Synthesis Telescope (MOST) at $843 \mathrm{MHz}$ and at 1.4, 2.4, 4.8 and $8.6 \mathrm{GHz}$ with the Australia Telescope Compact Array (ATCA) (Staveley-Smith et al. 1992). ATCA observations have been ongoing for $\sim 25$ years (Staveley-Smith et al. 1993; Gaensler et al. 1997; Ball et al. 2001; Manchester et al. 2002; StaveleySmith et al. 2007; Zanardo et al. 2010). An exponential increase in the flux density has been measured at all frequencies since day $\sim 5000$ after the explosion, which is likely due to an increasing efficiency of the acceleration process of particles by the shock front (Zanardo et al. 2010).

The morphology of the non-thermal radiation emitted by relativistic electrons accelerated in the remnant has been investigated using images at $9 \mathrm{GHz}$ since 1992 (Staveley-Smith et al. 1993) with a spatial resolution of 0.'5 achieved via maximum entropy super-resolution (Gaensler et al. 1997; $\mathrm{Ng}$ et al. 2008). These images have provided the first insight into the marked east-west asymmetry of the radio emission. As receivers operating in the $12 \mathrm{~mm}$ band $(16-26 \mathrm{GHz})$ were introduced at the ATCA in 2001, the first imaging observations at $18 \mathrm{GHz}$ were undertaken in 2003 July at an effective resolution of 0.45 (Manchester et al. 2005). Very long baseline interferometry (VLBI) observations of the SNR were successful in 2007 October (Tingay et al. 2009) and 2008 November ( $\mathrm{Ng}$ et al. 2011) at 1.4 and $1.7 \mathrm{GHz}$, respectively. These observations provided the first images with $\sim 0$ !' 1 resolution, but with low sensitivity and dynamic range. Nevertheless, the VLBI images captured the presence of small-scale structures in bright regions (Ng et al. 2011). At the same time, ATCA observations 
Table 1

Observing Parameters

\begin{tabular}{lccc}
\hline \hline Parameter & $18 \mathrm{GHz}$ & \multicolumn{2}{c}{$44 \mathrm{GHz}$} \\
\hline Date & 2011 Jan 26 & 2011 Jan 24 & 2011 Nov 19 \\
Day since explosion & 8738 & 8736 & 9036 \\
Center frequencies* $(\mathrm{GHz})$ & $16.963 \& 18.964$ & $43.026 \& 45.026$ \\
No. of antennas & 6 & 6 & 6 \\
Array configuration & $6 \mathrm{~A}$ & $6 \mathrm{~A}$ & $1.5 \mathrm{D}$ \\
Total observing time $(\mathrm{hr})$ & 9.18 & 9.68 & 9.82 \\
Averaged rms path length $(\mu \mathrm{m})$ & 274 & 236 & 198 \\
\hline
\end{tabular}

Note. ${ }^{*}$ CABB wideband mode on two interleaved $2.0 \mathrm{GHz}$ wide frequency bands, each with $2048 \times 1 \mathrm{MHz}$ channels.

at $36 \mathrm{GHz}$ in 2008 April and October resulted in high-dynamic range images with an angular resolution of 0.3 (Potter et al. 2009). The $36 \mathrm{GHz}$ images, in combination with the $9 \mathrm{GHz}$ observations at similar epochs, were used to construct the first spectral index image of the SNR, with a resolution of 0.'45. The resultant map provided the first glimpse into the spatial variations of the synchrotron spectral index across the remnant, and hinted at the possible association between flatter spectral indices and the bright sites in both lobes. After the ATCA upgrade in mid-2009 with the Compact Array Broadband Backend (CABB; Wilson et al. 2011), the remnant was imaged at higher frequencies. The first resolved image at $94 \mathrm{GHz}$ was produced from observations between 2011 June and August (Lakićević et al. 2012). Comparison of the $94 \mathrm{GHz}$ image with data at $9 \mathrm{GHz}$ yielded a low-resolution spectral index map, with a much larger region of flatter spectral index on the eastern lobe than on the western lobe.

This paper presents the first high-resolution image of SNR $1987 \mathrm{~A}$ at $44 \mathrm{GHz}$ and a new image at $18 \mathrm{GHz}$, both derived from ATCA observations performed in 2011. In Sections 2 and 3 we describe the imaging procedure and the resultant remnant morphology. In Section 4, we estimate the remnant expansion rate, by comparing the $44 \mathrm{GHz}$ data with the $36 \mathrm{GHz}$ data from 2008, while the new $18 \mathrm{GHz}$ data are compared to the 2003 datasets at 17 and $19 \mathrm{GHz}$. In Section 5 we assess the 18-44 GHz spectral index distribution, via different spectral mapping techniques. In Section 6, the comparison of the $44 \mathrm{GHz}$ image and contemporaneous X-ray and $\mathrm{H} \alpha$ observations, is discussed. In Section 7 we assess the likelihood of detecting at $44 \mathrm{GHz}$ the radiation emitted by a compact source located within the inner regions of the remnant.

\section{OBSERVATIONS}

SNR 1987A was observed at $44 \mathrm{GHz}$ with ATCA in 2011 January and November. The observations were performed on January 24 with the array in 6A configuration and on November 19 with the array in $1.5 \mathrm{D}$ configuration, with maximum baselines of $5939 \mathrm{~m}$ and $4439 \mathrm{~m}$, respectively. The observations were taken over $2 \times 2 \mathrm{GHz}$ bandwidth and centered on 43 and $45 \mathrm{GHz}$. The observations at $18 \mathrm{GHz}$ were performed on 2011 January 26 with the ATCA in 6A configuration, in two bands, each of $2 \mathrm{GHz}$ width, centered on 17 and $19 \mathrm{GHz}$. Atmospheric conditions were exceptional during both the $44 \mathrm{GHz}$ sessions with rms of the path length fluctuations below $250 \mu \mathrm{m}$, thus confirming that phase stability conditions at the ATCA can be met not only in winter but also in spring and summer nights (Middelberg et al. 2006). The rms of the path length fluctuations
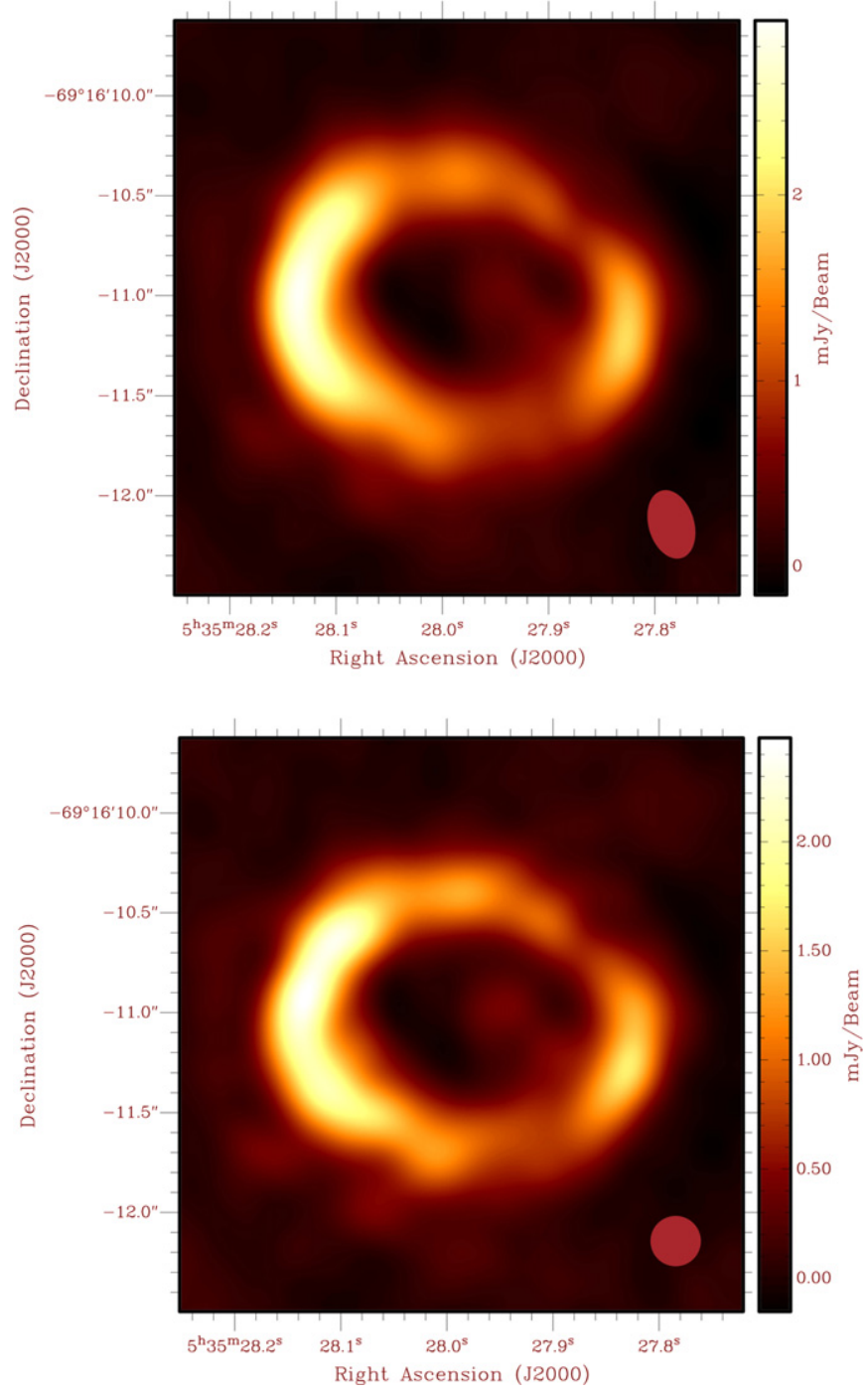

Figure 1. Top: diffraction-limited Stokes I continuum image of SNR 1987A at $44 \mathrm{GHz}$ made by combining observations performed with the ATCA on 2011 January 24 and 2011 November 16 . The beam size is $0.35 \times 0.23$ as plotted in the lower right corner. The off-source rms noise is $\sim 0.02 \mathrm{mJy}$ beam ${ }^{-1}$. Bottom: slightly super-resolved $44 \mathrm{GHz}$ image obtained using a 0.25 restoring circular beam (lower right corner).

was below $300 \mu \mathrm{m}$ during the $18 \mathrm{GHz}$ session. The parameters of the three observing sessions are listed in Table 1.

In all observations, the standard bandpass calibrator PKS B0637-752 was observed for 2 minutes every 90 minutes while the phase calibrator PKS 0530-727 was observed for 1.5 minutes every 6 minutes on the source. Uranus was used as the flux density calibrator at 43 and $45 \mathrm{GHz}$. At 17 and $19 \mathrm{GHz}$, we used PKS B1934-638 as the primary flux density calibrator. This is tied to Mars and has a stable flux density at $12 \mathrm{~mm}$ wavelength, as shown in Sault (2003). The absolute flux scale of both Mars and Uranus between 1 and $50 \mathrm{GHz}$ has been shown to have an uncertainty of 1\%-3\% (Perley \& Butler 2013). With good atmospheric conditions and good calibration, we estimate that the flux calibration accuracy at 18 and $44 \mathrm{GHz}$ is within $5 \%$. The observations were centered on R.A. $05^{\mathrm{h}} 35^{\mathrm{m}} 27^{\mathrm{s}} .975$, decl. $-69^{\circ} 16^{\prime} 11^{\prime \prime} .08$ (J2000), as in Potter et al. (2009).

The MIRIAD ${ }^{6}$ data reduction package was used to process all datasets. For the $44 \mathrm{GHz}$ observations, the task ATFIX was first

\footnotetext{
6 http://www.atnf.csiro.au/computing/software/miriad/
} 
Table 2

Image Parameters

\begin{tabular}{lcc}
\hline \hline Parameter & $44 \mathrm{GHz}$ & $18 \mathrm{GHz}$ \\
\hline Integrated flux density (mJy) & $40 \pm 2$ & $81 \pm 6$ \\
Restoring beam $\left(^{\prime \prime}\right)$ & $0.350 \times 0.225$ & $0.630 \times 0.474$ \\
Position angle $\left(^{\circ}\right)$ & 1.674 & 0.879 \\
rms noise (mJy/beam) & 0.023 & 0.088 \\
Dynamic range & $\sim 165$ & $\sim 176$ \\
\hline
\end{tabular}

used to apply corrections to the system temperatures, instrumental phases, and baseline lengths. The January and November datasets were imaged, CLEAN-ed (Högbom 1974), and selfcalibrated separately before being combined. In particular, the preliminary CLEAN model was constructed by using 400 iterations for the January dataset and 180 for the November dataset. Phase self-calibration was performed on the separate datasets and on the combined data, over a 2 minute solution interval. For imaging, a weighting parameter of robust $=0.5$ (Briggs 1995) was used. Deconvolution of the combined data was performed with the maximum entropy method (MEM; Gull \& Daniell 1978). The MEM model of the combined data led to a diffraction-limited image resolution of 0 '.35 $\times 0$ 0.23 with a moderate dynamic range $(\sim 165)$. The diffraction-limited image was then slightly super-resolved using a 0.25 circular beam. Figure 1 shows the combined Stokes $I$ continuum image derived from the January and November observations before and after super-resolution. The image yields an integrated flux density for the SNR of $40 \pm 2 \mathrm{mJy}$ at day 8886 after the explosion, where the rms error in the image and the flux density uncertainty are added in quadrature. In Stokes $Q, U$, and $V$ images, the source is not detected. The $3 \sigma$ upper limits to the flux densities are $60 \mu \mathrm{Jy} \mathrm{beam}^{-1}$ or $2 \%$ of the maximum Stokes $I$ flux density.

The data from the $18 \mathrm{GHz}$ observations were flagged then split into the separate observing bands until the imaging and deconvolution steps so as to account for frequency-dependent terms in the calibration step. Phase self-calibration was performed over a 2 minute solution interval, which gave sufficient signal-to-noise ratio $(\mathrm{S} / \mathrm{N})$. As for the reduction procedure of the $44 \mathrm{GHz}$ data, the robust weighting parameter was set at 0.5 and, after data deconvolution via MEM, the resulting image was restored with the diffraction-limited beam of half-maximum size $0.63 \times 00^{\prime} 47$. Because of the high $\mathrm{S} / \mathrm{N}$, a super-resolved image was also obtained by restoring the MEM model with a 0.25 circular beam, as Manchester et al. (2005) have shown that this degree of super-resolution gives reliable images. The diffraction-limited and super-resolved versions of the Stokes $I$ continuum $18 \mathrm{GHz}$ image of SNR 1987A, as derived at day 8738 since the explosion, are shown in Figure 2. The image gives an integrated flux density for the SNR of $81 \pm 6 \mathrm{mJy}$. The image parameters are summarized in Table 2.

\section{MORPHOLOGY OF THE RADIO EMISSION}

\section{1. $44 \mathrm{GHz}$ Morphology}

The emission at $44 \mathrm{GHz}$ appears to be mainly distributed in an elliptical ring, with brightness peaking on the eastern lobe. The east-west asymmetry of the radio emission is a characteristic of SNR 1987A, which has emerged since the first ATCA images (Gaensler et al. 1997) and has been monitored via the super-resolved ATCA images at $9 \mathrm{GHz}$ ( $\mathrm{Ng}$ et al. 2008, hereafter N08). This asymmetry has been confirmed in ATCA
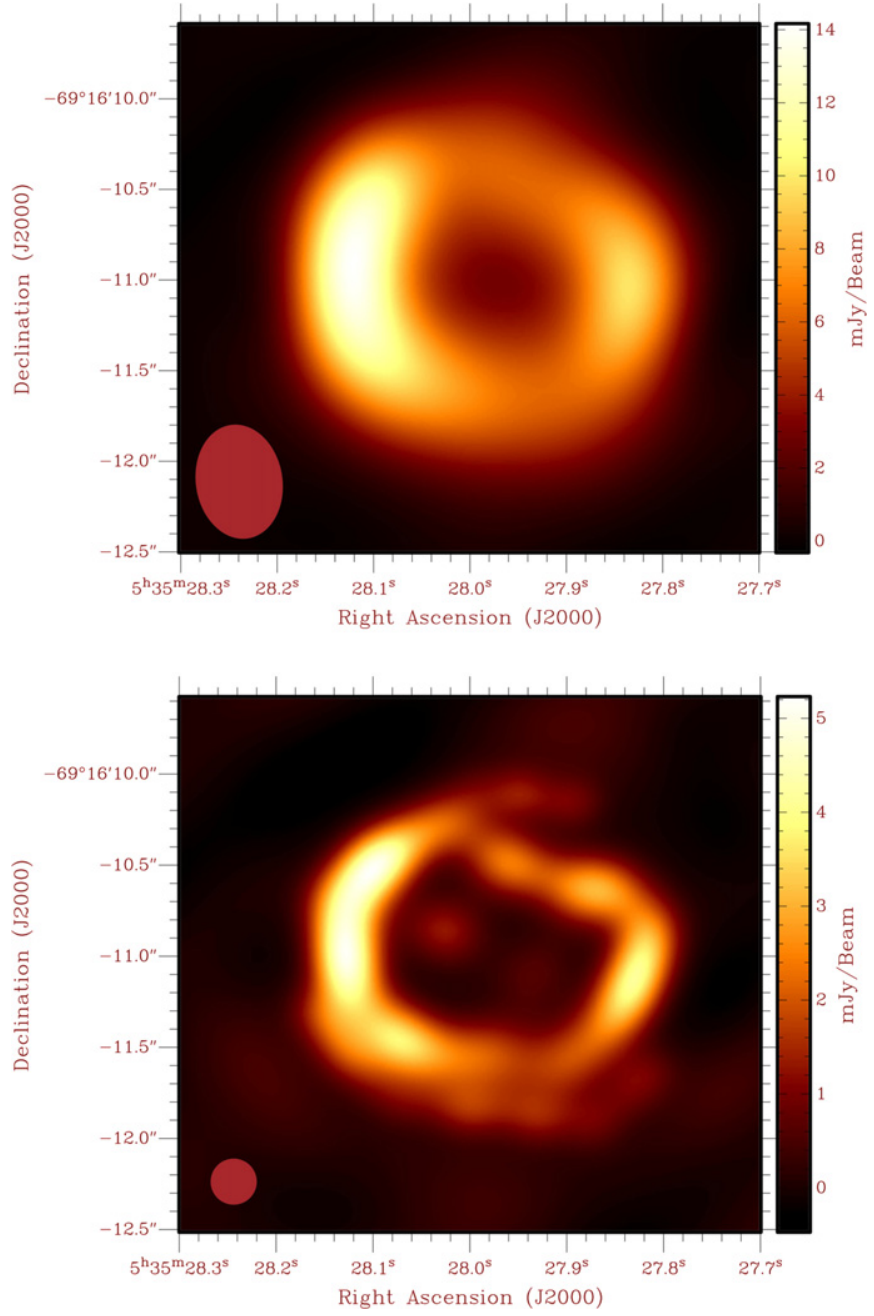

Figure 2. Top: diffraction-limited Stokes $I$ continuum image of SNR 1987A at $18 \mathrm{GHz}$ from observations performed on 2011 January 26 using the ATCA. The beam size is $00^{\prime \prime} 63 \times 0.0^{\prime \prime} 47$ as plotted in the lower left corner. The off-source rms noise is $\sim 90 \mu \mathrm{Jy}$ beam $^{-1}$. Bottom: super-resolved $18 \mathrm{GHz}$ image obtained using a 0.25 restoring circular beam (lower left corner).

observations at 18, 36, and $94 \mathrm{GHz}$ (Manchester et al. 2005; Potter et al. 2009; Lakićević et al. 2012). Slices through the $44 \mathrm{GHz}$ image at six position angles are shown in Figure 3. The brightness of the eastern lobe peaks between the radial profiles at $60^{\circ}$ and $90^{\circ}$, while the ratio of the profile maxima corresponding to the brightness peaks of the eastern and western lobe is $\sim 1.5$. Overall, the eastern half of the image appears significantly brighter than the western one, with a ratio of $\sim 1.6$ between the integrated flux densities of the two regions, i.e., east and west of the geometric center, approximately located $\sim 75$ mas east of the VLBI position of the SN (Reynolds et al. 1995; R.A. 05 $35^{\mathrm{m}} 27^{\mathrm{s}} .968$, decl. $-69^{\circ} 16^{\prime} 11^{\prime \prime}$.09 (J2000)). This emission ratio is higher than the $\sim 1.4$ obtained at $9 \mathrm{GHz}$ (N08) and the $\sim 1.3$ measured at $36 \mathrm{GHz}$ (Potter et al. 2009), via fitting the images with an equatorial belt torus characterized by a west-east linear gradient of the brightness distribution (see N08).

The profiles also show a possible third peak slightly west of the center of the remnant, which reaches maximum in the $120^{\circ}$ slice, at $47 \%$ of the brightness peak of the western lobe. The overall emission from this central region is $1.4 \pm 0.2 \mathrm{mJy}$, thus making $\sim 4 \%$ of the total integrated flux density. 

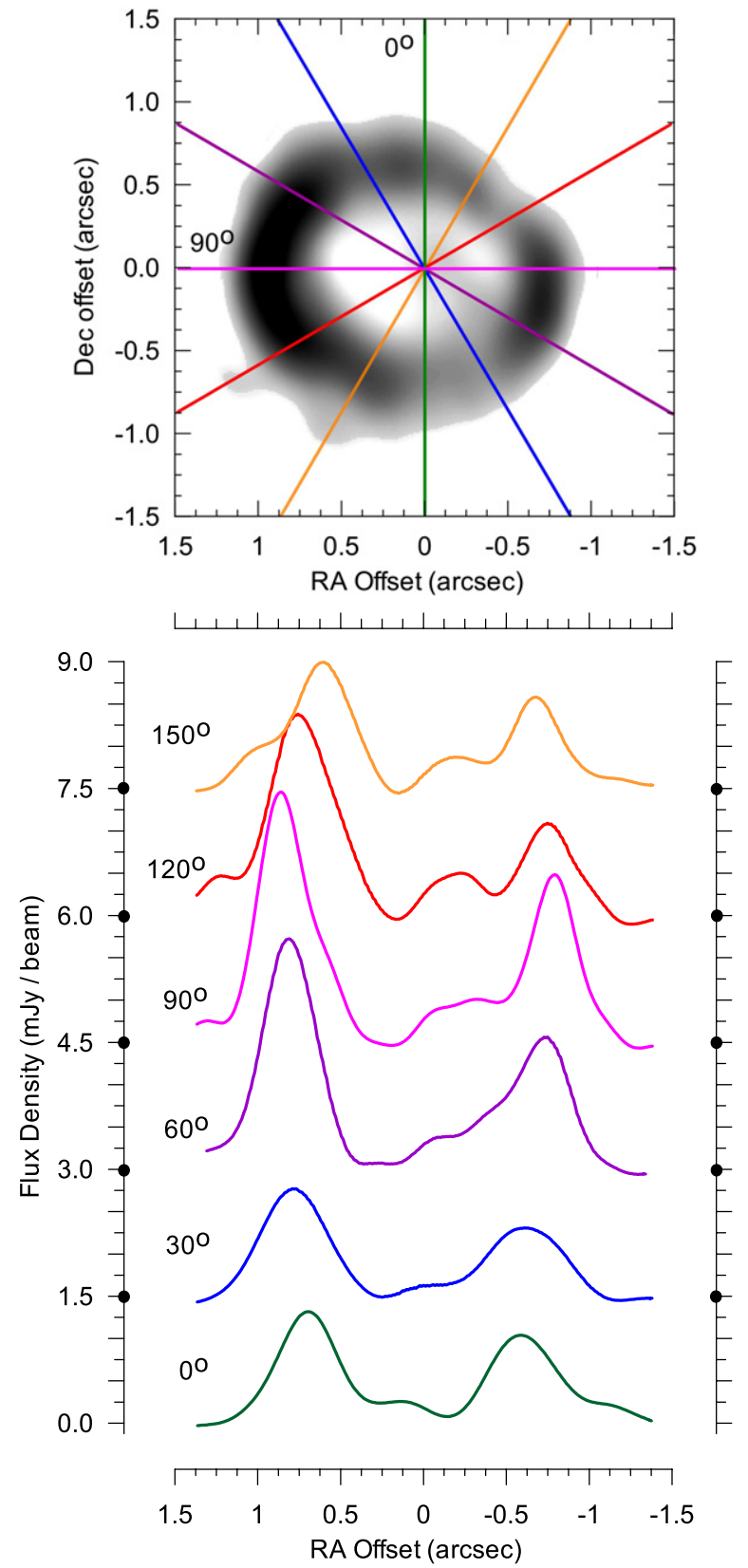

Figure 3. Radial slices through the diffraction-limited $44 \mathrm{GHz}$ image at six position angles. Black dots on vertical axes indicate the position of the zero for each slice. The offset is radial from the VLBI position of SN 1987A as determined by Reynolds et al. (1995; R.A. $05^{\mathrm{h}} 35^{\mathrm{m}} 27^{\mathrm{s}} .968$, decl. $-69^{\circ} 16^{\prime} 11^{\prime \prime} .09$ (J2000)), and is positive toward east and/or north.

(A color version of this figure is available in the online journal.)

In Figure 4, the $44 \mathrm{GHz}$ image is compared to the 2008 observations at $36 \mathrm{GHz}$ (see Table 3 ). The remnant expansion over the 4 year time frame is noticeable from the contours of the peak flux density at identical levels. A total integrated flux density of $23.6 \pm 5.2 \mathrm{mJy}$ is derived by scaling the flux density of the $36 \mathrm{GHz}$ image to that associated with the remnant at $44 \mathrm{GHz}$ at the same epoch, as $\alpha=-0.68$ results at day 7815 from the spectral index function that fits observations between 1.4 and 8.6 GHz (see Figure 7 in Zanardo et al. 2010). This leads to a yearly flux density increase of $24 \pm 6 \%$, which is higher than the $17 \pm 8 \%$ per year derived at $8.6 \mathrm{GHz}$ by Zanardo et al. (2010), from monitoring observations between Years 20 and 21 .

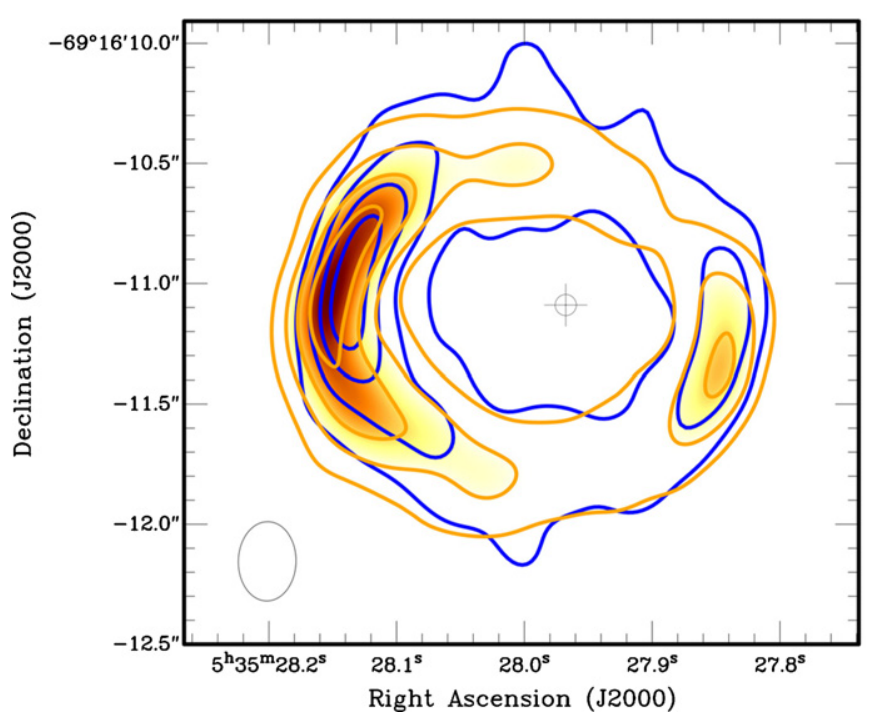

Figure 4. Comparison between the $44 \mathrm{GHz}$ image and that derived from ATCA observations at $36 \mathrm{GHz}$ performed in 2008 (Potter et al. 2009). The $44 \mathrm{GHz}$ image is restored with the $0 . \prime 33 \times 0,24$ beam that characterizes the diffractionlimited image at $36 \mathrm{GHz}$, which is set at P.A. -1.3 (Potter et al. 2009; Table 3). The contours at levels $25 \%-85 \%$ of the peak flux density, in step of $10 \%$, are in blue for the $36 \mathrm{GHz}$ image and in orange for the $44 \mathrm{GHz}$ image. To highlight the morphological differences of the brightest sites in the two images, the $44 \mathrm{GHz}$ contours at $45 \%, 65 \%$ and $85 \%$ of the peak flux density, are filled in yellow, orange and brown. The cross-hair symbol marks the VLBI position of SN 1987A (Reynolds et al. 1995).

(A color version of this figure is available in the online journal.)

In Figure 5, the $44 \mathrm{GHz}$ image is compared with that derived by fitting the N08 inclined-torus model to the $9 \mathrm{GHz}$ observations performed on 2011 April 22 (C.-Y. Ng et al. 2013, in preparation). The fitted model has a radius of $0^{\prime \prime} .917$, which surpasses by $\sim 67$ mas that used to fit the $36 \mathrm{GHz}$ image in 2008 (Potter et al. 2009). The flux of the $9 \mathrm{GHz}$ model is scaled to that corresponding at $44 \mathrm{GHz}$, via the spectral index $\alpha=-0.74$, which is extrapolated from contemporaneous ATCA observations spanning from 1.4 to $94 \mathrm{GHz}$ (see Figure 6). The resultant model image, as shown in Figure 5, has a total integrated flux density of $40 \pm 1 \mathrm{mJy}$ and the restoring beam of the diffraction-limited image at $44 \mathrm{GHz}$. The residual between the model and the actual visibilities demonstrates that the model matches the observations along the profile at P.A. $90^{\circ}$, where the ratio of the brightness peaks between the two lobes equals the $\sim 1.6$ measured in the observations. The residual visibilities peak on the eastern lobe at P.A. $30^{\circ}$ and $150^{\circ}$, since the new observations exhibit more extended bright regions than what reproduced by the linear gradient of the modeled flux distribution. The linear gradient assumption also constrains the ratio of the integrated flux density over the eastern and western halves of the image, to $\sim 1.3$. Therefore, the N08 model, which fits well the radio remnant as seen at $9 \mathrm{GHz}$, might not be applicable to the large-scale emission morphology that emerges from the $44 \mathrm{GHz}$ observations.

\section{2. $18 \mathrm{GHz}$ Morphology}

In the diffraction-limited image, the emission at $18 \mathrm{GHz}$ looks primarily ring-shaped, while a secondary structure seems to emerge from the super-resolved image (see Figure 2). Two armlike features appear to stem from the northern and southern edges of the major ellipsoidal structure of the emission, peaking at P.A. $0^{\circ}$, and extending toward the western side of the remnant. 

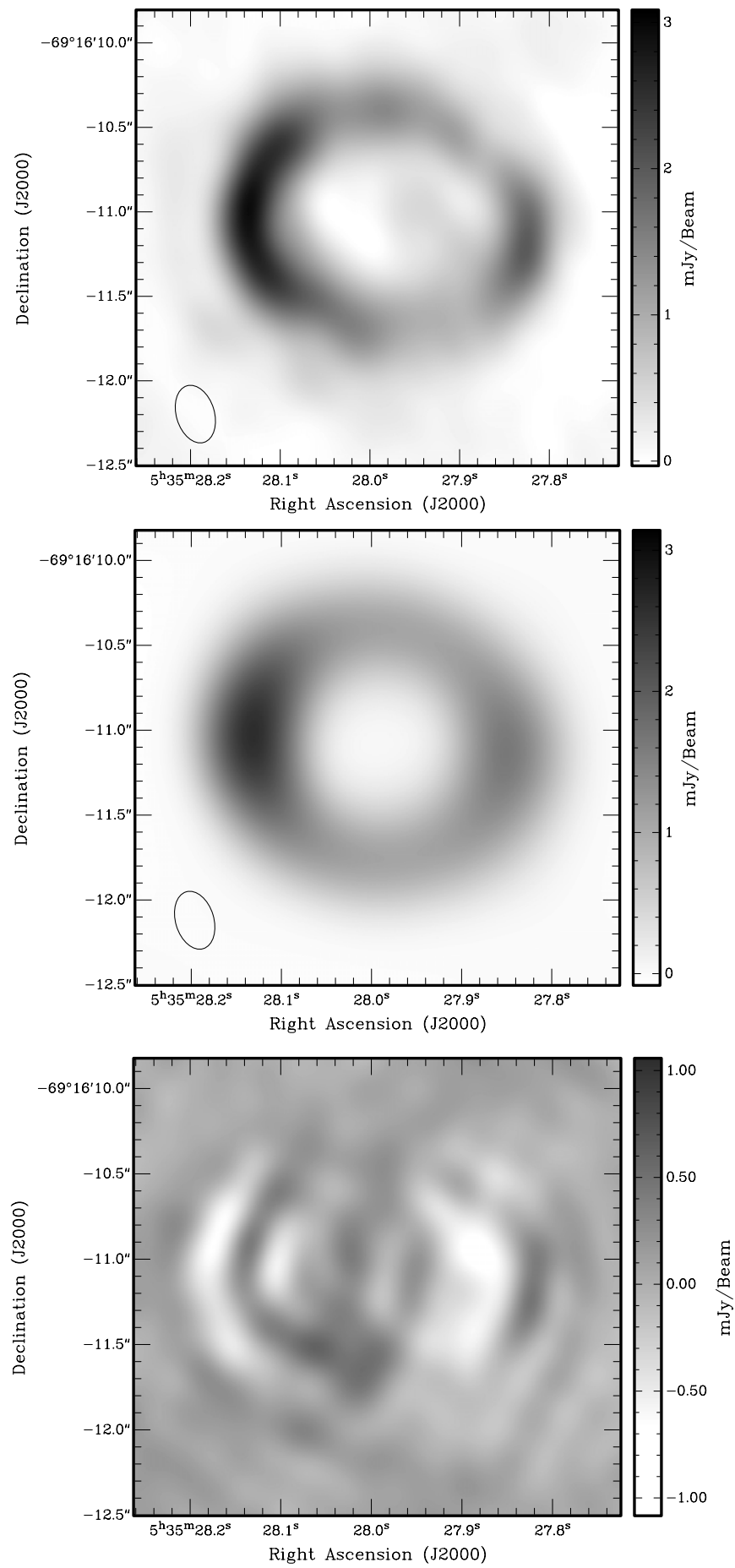

Figure 5. Comparison of the diffraction-limited $44 \mathrm{GHz}$ image (top), as derived from ATCA observations performed in 2011 January and November, with the image generated with the Fourier-domain model (center) developed by $\mathrm{Ng}$ et al. (2008) to fit the SNR observations at $9 \mathrm{GHz}$. The model used in the comparison fits the $9 \mathrm{GHz}$ observations performed in 2011 April 22 (C.-Y. Ng et al. 2013, in preparation), and has a radius of $0{ }^{\prime \prime} 917$. To match the total flux of the observations at $44 \mathrm{GHz}$, the model flux density has been scaled using a spectral index $\alpha=-0.74$ (see Figure 6). Bottom: dirty map of the residual visibilities obtained by subtracting the model from the $44 \mathrm{GHz}$ observations. No deconvolution has been applied to the residual.

While these features suggest a double-ring formation within the remnant, they might more likely be noise artifacts of the superresolution process.

In terms of remnant asymmetry, at $18 \mathrm{GHz}$ the radial profiles in Figure 7 show that the asymmetry peaks on the eastern lobe

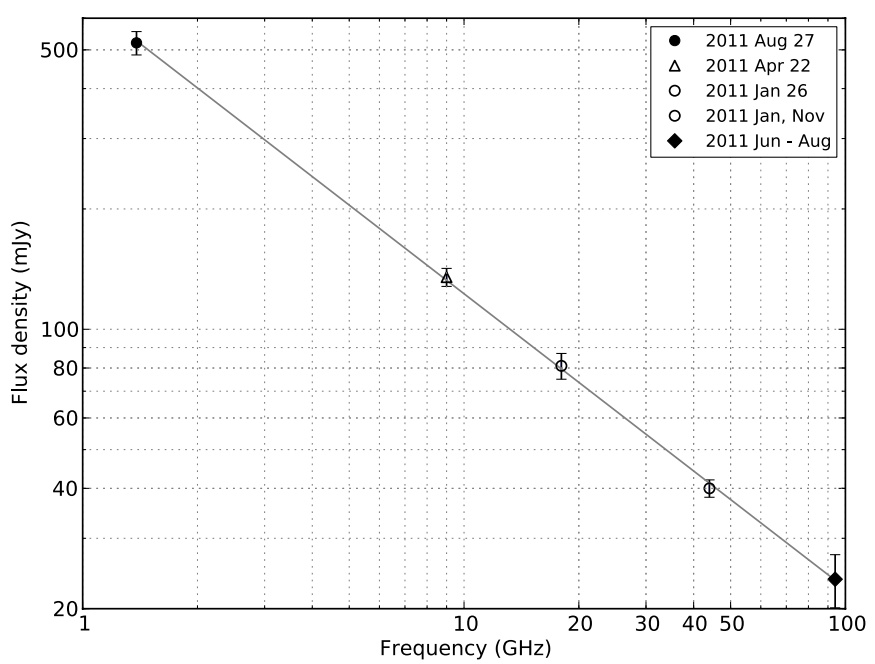

Figure 6. Spectral index of SNR 1987A as determined from ATCA observations performed in 2011. In detail, the spectral index is determined from the powerlaw fit to the flux densities measured at five frequencies: $1.4 \mathrm{GHz}$ (G. Zanardo et al. 2003, in preparation; solid circle), $9 \mathrm{GHz}$ (C.-Y. Ng et al. 2013, in preparation; hollow triangle), 18 and $44 \mathrm{GHz}$ (this paper; hollow circle), and $94 \mathrm{GHz}$ (Lakićević et al. 2012; solid diamond). The fit yields $\alpha=-0.74 \pm 0.01$.

Table 3

Pre-CABB ATCA Images at 18 and $36 \mathrm{GHz}$

\begin{tabular}{lcc}
\hline \hline Parameter & $18 \mathrm{GHz}^{\mathrm{a}}$ & $36 \mathrm{GHz}^{\mathrm{b}}$ \\
\hline Observing session & $2003 \mathrm{Jul} 31$ & $2008 \mathrm{Apr} 25 \&$ Oct $7,8,12$ \\
Day since explosion & 6003 & $7815^{\mathrm{c}}$ \\
Center frequencies $(\mathrm{GHz})$ & $17.3 \& 19.6$ & $34.9 \& 37.4$ \\
Flux density (mJy) & $27 \pm 4$ & $27 \pm 6$ \\
Restoring Beam (") & $0.45 \times 0.39$ & $0.33 \times 0.24$ \\
Position Angle $\left(^{\circ}\right)$ & $2^{\circ}$ & -1.3 \\
Super-resolution $\left({ }^{\prime \prime}\right)$ & 0.25 & $\cdots$ \\
\hline
\end{tabular}

Notes.

${ }^{a}$ See Manchester et al. (2005).

${ }^{\mathrm{b}}$ See Potter et al. (2009).

${ }^{c}$ Average date of the combined datasets.

between P.A. $60^{\circ}$ and $90^{\circ}$. The asymmetry ratio between the eastern and western brightness peaks is $\sim 1.4$, and a similar ratio is obtained from the ratio between the flux density integrated over the eastern and western halves of the image. This value matches the asymmetry ratio derived by N08 from 1992-2008 data at $9 \mathrm{GHz}$.

In Figure 8, the super-resolved image is compared to that derived from previous ATCA observations at $18 \mathrm{GHz}$ performed on 2003 July 31, which was also super-resolved to 0.25 (Manchester et al. 2005; Table 3). The morphology similarities between the 2003 and 2011 images emphasize the significant expansion of the remnant over 8 years. Slices through the 2011 image indicate that the central emission varies from $20 \%$ to $24 \%$ of the brightness on the eastern lobe. This figure is consistent with the 2003 observations, where the central emission was estimated $\sim 20 \%$ of the average ring intensity (Manchester et al. 2005).

The integrated flux density of the 2003 image, as derived from the combination of the data at 17.3 and $19.6 \mathrm{GHz}$, was $27 \pm 4 \mathrm{mJy}$, which is lower than what expected from an extrapolation of the radio spectrum. This value leads to a threefold increase in flux from day 6003 to 8738, i.e., a yearly increase rate of $27 \pm 5 \%$. This increase rate exceeds 

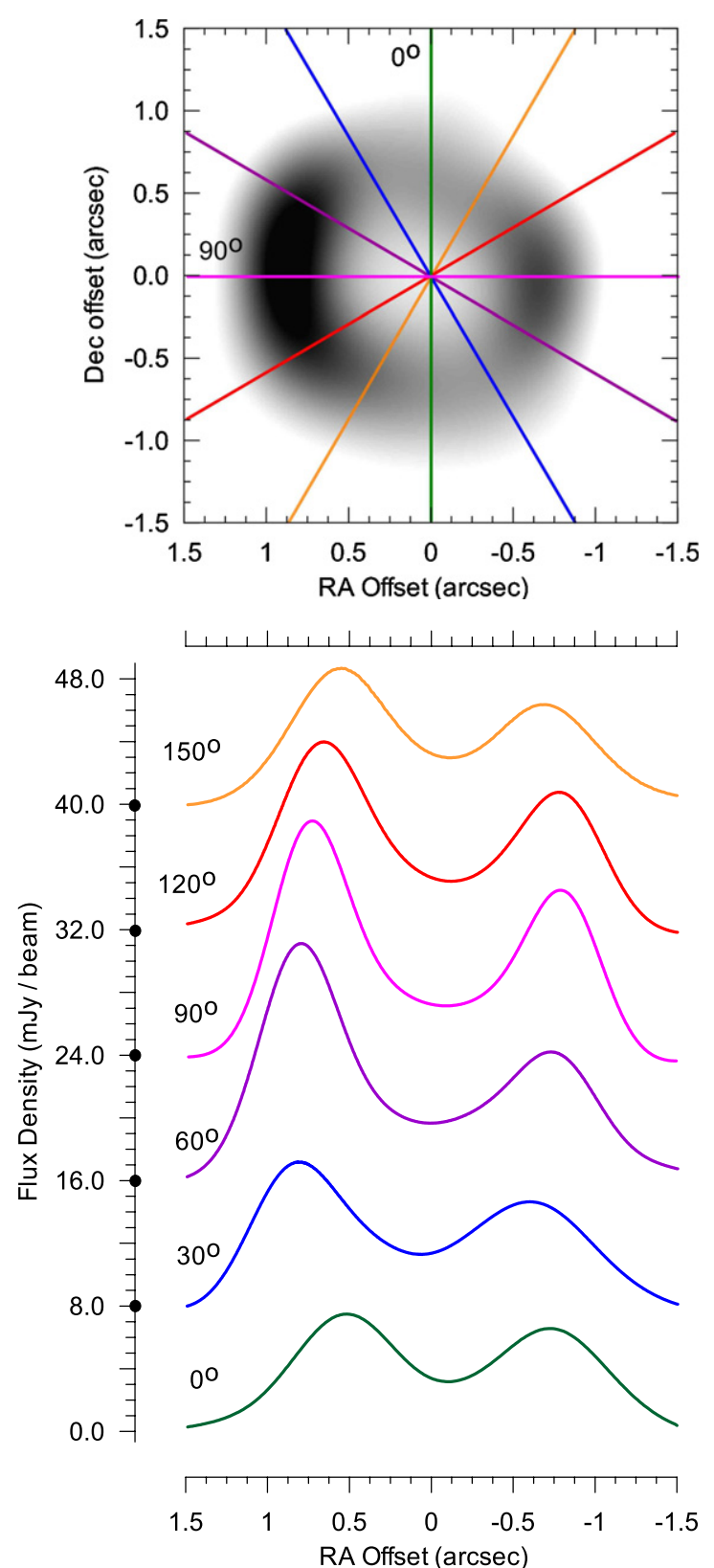

Figure 7. Radial slices through the diffraction-limited $18 \mathrm{GHz}$ image at six position angles. Black dots on vertical axes indicate the position of the zero for each slice. The offset is radial from the VLBI position of SN 1987A as determined by Reynolds et al. (1995), and is positive toward east and/or north. (A color version of this figure is available in the online journal.)

the $17 \pm 8 \%$ per year derived at $8.6 \mathrm{GHz}$ by Zanardo et al. (2010), for data between Years 20 and 21 since the SN.

\section{REMNANT EXPANSION}

From 1987 to 1992 , i.e., in the early stages of the SNR, the remnant had an expansion velocity of $\sim 30,000 \mathrm{~km} \mathrm{~s}^{-1}$ (Gaensler et al. 1997). When the radio emission re-emerged, this was followed by a drastic deceleration to $3000 \mathrm{~km} \mathrm{~s}^{-1}$ (Gaensler et al. 1997). From 1992 to 2008, the expansion has been determined from the radius of the $9 \mathrm{GHz}$ images fitted via a torus model, with a resultant velocity of $4000 \pm 400 \mathrm{~km} \mathrm{~s}^{-1}$ from $\sim$ day 1800 to day 7620 (N08).

In Figure 9, the expansion of the remnant at 18 and $44 \mathrm{GHz}$ is estimated as the change over time of the distance between

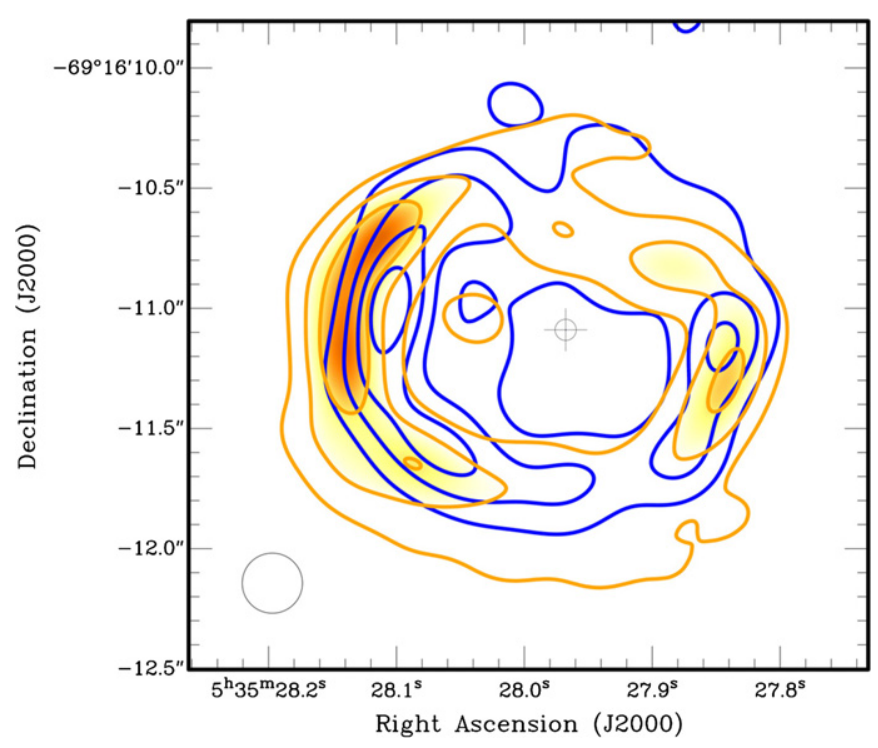

Figure 8. Comparison between the new $18 \mathrm{GHz}$ image and that derived from ATCA observations at $18 \mathrm{GHz}$ performed in 2003 (Manchester et al. 2005). Both images are super-resolved with a 0.25 circular beam. The contours at levels $25 \%-85 \%$ of the peak flux density, in step of $10 \%$, are in blue for the 2003 image and in orange for the new image. To highlight the morphological differences of the brightest sites in the two images and the significant expansion of the remnant since 2003 , the contours of the new image at $45 \%, 65 \%$, and $85 \%$ of the peak flux density, are filled in yellow, light and dark orange. The cross-hair symbol marks the VLBI position of SN 1987A (Reynolds et al. 1995). (A color version of this figure is available in the online journal.)

the VLBI position of the SN (Reynolds et al. 1995) and the mean position of the peak of brightness for the eastern and western lobes, as derived from the emission profile at P.A. $90^{\circ}$ centered on the $\mathrm{SN}$ coordinates. Comparison between observations at $18 \mathrm{GHz}$ at days 6003 and 8738, gives a velocity of $4100 \mathrm{~km} \mathrm{~s}^{-1}$ (blue line), while the comparison between the 36 and $44 \mathrm{GHz}$ datasets, within the 2008-2011 time frame, leads to a velocity of $3900 \mathrm{~km} \mathrm{~s}^{-1}$ (purple line). An identical result of $3900 \pm 300 \mathrm{~km} \mathrm{~s}^{-1}$ is obtained if the 2011 dataset at $18 \mathrm{GHz}$ is included in the fit of data between 2008 and 2011 since the $18 \mathrm{GHz}$ measurements align with those at 36 and $44 \mathrm{GHz}$. All fits are consistent with the $9 \mathrm{GHz}$ results.

With reference to the SN position, it should be noted that both the datasets at $18 \mathrm{GHz}(2003-2011)$ and the datasets at $36 \mathrm{GHz}(2008)$ and $44 \mathrm{GHz}$ (2011), show an asymmetric expansion of the remnant, with a larger increase of the distances between the SN position and that of the brightness peak on the eastern lobe compared to the western side. The asymmetry in the expansion has been noted since the images at $9 \mathrm{GHz}$ in early epochs (Gaensler et al. 2007), where an asymmetry in the initial expansion of the $\mathrm{SN}$ ejecta has been proposed as the explanation for the fact that the eastern lobe is located further than the western one from the SN site. In the case of the $18 \mathrm{GHz}$ datasets, an expansion velocity of $5900 \mathrm{~km} \mathrm{~s}^{-1}$ is derived for the eastern lobe, while $2300 \mathrm{~km} \mathrm{~s}^{-1}$ is the expansion velocity of the western brightness peak. Similarly, in the datasets at 36 and $44 \mathrm{GHz}$, the eastern expansion velocity is $6300 \mathrm{~km} \mathrm{~s}^{-1}$ while the western is $1600 \mathrm{~km} \mathrm{~s}^{-1}$. Averaging the values, the expansion velocity of the remnant western lobe is $1900 \pm 400 \mathrm{~km} \mathrm{~s}^{-1}$, while that of the eastern lobe is $6100 \pm 200 \mathrm{~km} \mathrm{~s}^{-1}$. While these values are affected by some uncertainty in identifying the location of the remnant with respect to the VLBI coordinates of the SN site, the results from the 18,36 , and $44 \mathrm{GHz}$ data imply that the expansion velocity of the eastern lobe is significantly larger than 


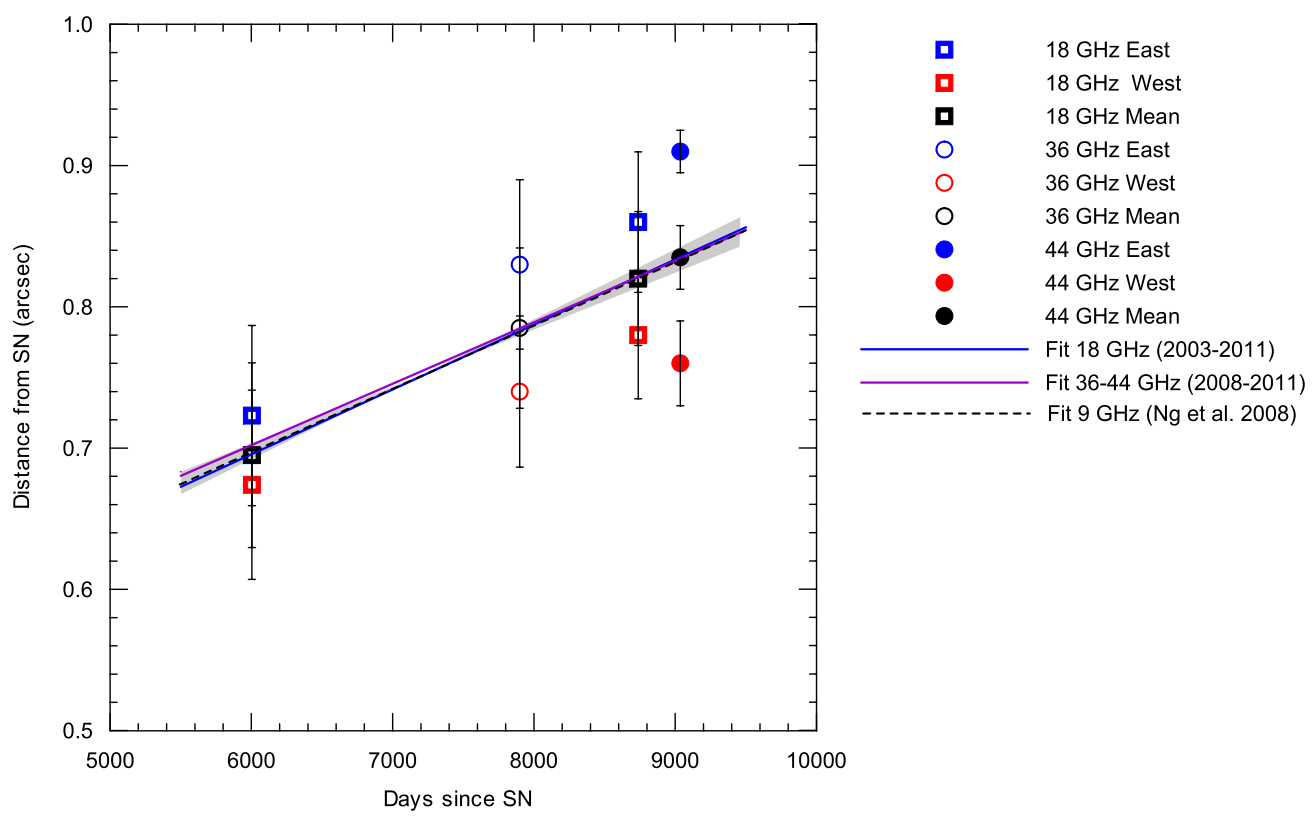

Figure 9. Remnant expansion from images at $18 \mathrm{GHz}$ on day 6003 (Manchester et al. 2005) and day 8738 and at $36 \mathrm{GHz}$ on day 7815 (Potter et al. 2009) and $44 \mathrm{GHz}$ on day 8886 (this paper). The expansion is estimated from the distance between the VLBI position of the SN (Reynolds et al. 1995) and the position of the peak of brightness for the eastern and western lobes, as derived from the P.A. $90^{\circ}$ emission profiles centered on the SN coordinates. The blue symbols are associated with the brightness peaks on the eastern lobes, while the red ones represent the distance between the SN position and the brightness maximum in the western lobe. Mean values derived for each pair of measurements are represented by the black symbols. The blue line is the linear fit of the measurements at $18 \mathrm{GHz}$ from 2003 to 2011 , while the purple line only fits the 36 and $44 \mathrm{GHz}$ within 2008 and 2011. For data between 2008 and 2011, the derived expansion velocity is $3900 \pm 300 \mathrm{~km} \mathrm{~s}^{-1}$. As a comparison with the expansion rate derived by $\mathrm{Ng}$ et al. (2008), the dashed black line is the slope of the linear fit of radius measurements extracted from the 1992-2008 super-resolved images at $9 \mathrm{GHz}$ fitted with a torus model, the error associated with the fit is represented by the gray-filled area.

(A color version of this figure is available in the online journal.)

that of the western lobe. Therefore, a marked asymmetry of the remnant emerges not only in terms of brightness distribution of the radio emission but also in terms of remnant expansion.

\section{SPECTRAL INDEX MEASUREMENTS}

Spectral index maps allow the examination of the spatial variations of the spectral index, $\alpha$ (where $S \propto v^{\alpha}$ ), across the remnant and, therefore, of the electron acceleration processes associated with the propagation of the blast wave. The 2011 observations at 18 and $44 \mathrm{GHz}$ have been used to derive a map of the distribution of the spectral index in the SNR. To match the $u-v$ coverage of the $18 \mathrm{GHz}$ observations, the visibilities from baselines greater than $400 \mathrm{k} \lambda$ were filtered out in the $44 \mathrm{GHz}$ datasets. At both frequencies, the images have been derived using an identical reduction procedure. In particular, identical weighting parameters, a deconvolution algorithm, and phase self-calibration iterations were applied to both datasets. It should be noted that the self-calibration technique, while improving the image resolution, removes all absolute positional information. Therefore, the astrometry of each image was compared with that prior to self-calibration and the images were shifted to align with recent VLBI observations of the remnant (G. Zanardo et al. 2003 , in preparation). This process allowed us to accurately place the position of the remnant with respect to the VLBI position of the SN mentioned in Section 3. The aligned images were then restored with a 0.4 circular beam and regridded at a pixel scale of 3 mas.

\subsection{Methods for Measuring the Spectral Index}

To verify that any claimed spectral index variations have not been artificially generated by the data reduction and/or are tied to a particular spectral mapping technique, the spectral index distribution within the remnant is reconstructed via three different methods: (1) spectral tomography $\left(\alpha_{t}\right)$, (2) flux ratio $\left(\alpha_{S}\right)$, and (3) temperature-temperature (or $\left.T-T\right)$ plots $\left(\alpha_{T T}\right)$.

With the method of spectral tomography (Katz-Stone \& Rudnick 1997), a difference image, $I_{t}$, is calculated by scaling the $44 \mathrm{GHz}$ image by a trial spectral index, $\alpha_{t}$, and subtracting it from the $18 \mathrm{GHz}$ image, as follows:

$$
I_{t}\left(\alpha_{t}\right) \equiv I_{18}-\left(\frac{v_{18}}{v_{44}}\right)^{\alpha_{t}} I_{44}
$$

where $I_{18}$ and $I_{44}$ are the images at frequency $v_{18}$ and $v_{44}$, respectively. When $\alpha_{t}$ reaches the actual spectral index of a particular feature, the feature vanishes in the local background of the difference image. On the other hand, if a component has spectral index greater or smaller than $\alpha_{t}$, the difference image appears with a distinctively positive or negative residual compared to the local background, as this component will get over- or under-subtracted.

The second method used to construct a spectral index map is that of direct image division, being $\alpha_{S}=$ $\log \left(S_{18} / S_{44}\right) / \log (18 / 44)$. As this method is sensitive to variations in background/foreground emission that might cause spatial changes in the observed spectral index, it is not usually the preferred method to investigate the spectral index distribution over large SNRs (Anderson \& Rudnick 1993).

To validate the results from the flux density ratio $S_{18} / S_{44}$, the spectral variations over small regions within the remnant are also assessed from the flux slope $m$, where $S_{18}=m S_{44}+q$. This approach was first applied by Costain (1960) and Turtle et al. (1962) to data of very low angular resolution, where instead of fluxes, the brightness temperatures, $T_{b} \propto v^{-(\alpha+2)}$, are plotted at two frequencies, and the spectral index is derived 
from the best fitting line of the plotted points (thus referred to as a temperature-temperature or $T-T$ plot).

The slope of the best-fitting line to the $T_{b}$ points derived for each pixel of the images at 18 and $44 \mathrm{GHz}\left(T_{18}\right.$ and $\left.T_{44}\right)$, within a specific region, yields the spectral index $\alpha_{T T}$ of that region. While small differences in the background levels cause spreading of the plotted $T-T$ points, because of the intrinsic spatial averaging of the linear regression method, the regional $\alpha_{T T}$ values are less affected than $\alpha_{S}$ by zero-level data (Leahy \& Roger 1991; Zhang et al. 1997).

\subsection{Interpretation of Spectral Index Variations}

Understanding the spatial variation of the spectral index and emission across young SNRs is important to investigate the structure of the expanding shock. The spectral index can be used to probe the compression ratio of the shock on a local scale. In turn, the emission probes cosmic ray and magnetic field density. Combined, these provide the basis for investigating the nature of shock acceleration processes.

In the tomography gallery in Figure 10, spectral index variations are investigated in the range between $-1.4 \leqslant \alpha_{t} \leqslant$ -0.3 , with increment $\delta \alpha_{t}=0.1$. It can be seen that the spectral index distribution across the remnant is not uniform. In detail, the inner areas of the remnant located along P.A. $\sim 30^{\circ}\left(210^{\circ}\right)$ appear to match the gray background for $-0.6 \leqslant \alpha_{t} \leqslant-0.3$, with $\alpha_{t} \sim-0.4$ in the inner part of the northern region, and $\alpha_{t} \sim-0.5$ in the inner part of the feature located slightly SW of the SN position. The spectral index gradually steepens toward the edges, with the northern and southern regions becoming over-subtracted at $\alpha_{t} \sim-0.7$ and $\alpha_{t} \sim-1.0$, respectively. For the bright regions on the eastern lobe of the remnant, sites within the contours at $90 \%$ of the peak flux density (see Figure 10) primarily match the gray background for $\alpha_{t} \sim-0.8$. Within the $60 \%$ contours, $\alpha_{t}$ changes from -0.7 (southern part), to -0.8 (central region) and -0.9 (northern region). The entire bright area on the eastern lobe is over-subtracted for $\alpha_{t} \sim-1.1$. On the western lobe, sites within the $60 \%$ contours have a wider range than on the eastern lobe, as $\alpha_{t}$ varies from -1.1 to -0.7 with a north-south gradient. Southern regions in the tomography maps disappear into the background for $-0.8 \leqslant \alpha_{t} \leqslant-0.7$, with larger errors on sites close to the edges of the $10 \%$ flux density contour of the $44 \mathrm{GHz}$ image. Larger spectral index variations can be seen in the N-NW sites, with $-0.9 \leqslant \alpha_{t} \leqslant-0.6$. The sites that can be associated with spectral index $-1.4 \leqslant \alpha_{t} \leqslant-1.2$ correspond to flux density at $44 \mathrm{GHz}$ close to zero and, therefore, are more affected by error in the image subtraction.

The spectral index map, as derived from the direct division of the images at 44 and $18 \mathrm{GHz}$, is shown in Figure 11. It can be seen that the spectral indices primarily vary in the range $-0.9 \leqslant \alpha_{S} \leqslant-0.7$. In particular, on the eastern lobe, within the $90 \%$ contours of the peak flux density at both 18 and $44 \mathrm{GHz},-0.9 \leqslant \alpha_{S} \leqslant-0.7$, while slightly steeper values, $-1.0 \leqslant \alpha_{S} \leqslant-0.8$, correspond with the western brightest sites. On both lobes, near the northern edge of the $60 \%$ contours, the spectral indices peak at $\sim-0.9$ on the eastern sites and $\sim-1.1$ on the western ones. Flatter spectral indices $\left(-0.6 \leqslant \alpha_{S} \leqslant-0.3\right)$ can be located within the central and northern regions of the remnant at $\sim$ P.A. $30^{\circ}$.

It is noted that the regions at the edges of the $\alpha_{S}$ map, i.e., $\alpha_{S} \lesssim-1.2$ (in dark-blue and black) and $\alpha_{S} \gtrsim-0.3$ (in lightblue and white) are likely affected by higher uncertainty, since these values are associated with lower $\mathrm{S} / \mathrm{N}$ ratios in either
Table 4

Regional Spectral Indices

\begin{tabular}{lcccc}
\hline \hline Region $^{\mathrm{a}}$ & $\alpha_{T T}{ }^{\mathrm{b}}$ & $\alpha_{S_{\mu}}{ }^{\mathrm{c}}$ & $\Delta \alpha^{\mathrm{d}}$ & $\begin{array}{c}\Delta \alpha / \alpha_{S_{\mu}} \\
(\%)\end{array}$ \\
\hline N1 & $-0.88 \pm 0.13$ & $-0.67 \pm 0.05$ & 0.21 & 31 \\
N2 & $-0.65 \pm 0.25$ & $-0.71 \pm 0.05$ & 0.06 & 8 \\
E1 & $-0.91 \pm 0.07$ & $-0.79 \pm 0.06$ & 0.12 & 16 \\
E2 & $-0.80 \pm 0.06$ & $-0.66 \pm 0.05$ & 0.14 & 22 \\
S1 & $-0.85 \pm 0.09$ & $-0.73 \pm 0.05$ & 0.12 & 16 \\
S2 & $-0.72 \pm 0.09$ & $-0.85 \pm 0.06$ & 0.13 & 15 \\
W1 & $-0.76 \pm 0.16$ & $-1.09 \pm 0.08$ & 0.33 & 31 \\
W2 & $-0.80 \pm 0.11$ & $-0.75 \pm 0.05$ & 0.05 & 6 \\
C1 & $-0.52 \pm 0.18$ & $-0.80 \pm 0.06$ & 0.28 & 35 \\
\hline
\end{tabular}

Notes.

a The regions selected for the $T-T$ plots are designated in Figure 13 .

b The errors on $\alpha_{T T}$ are the combination of the $1 \sigma$ error on the slope of the linear fit and the uncertainty in the flux calibration.

${ }^{\mathrm{c}}$ The median spectral index, $\alpha_{S_{\mu}}$, is derived by fitting a Gaussian to the histogram of the $\alpha_{S}$ values in the region (see Figure 14). The errors are derived from the flux calibration uncertainty.

${ }^{\mathrm{d}} \Delta \alpha=\left|\alpha_{\mathrm{TT}}-\alpha_{S_{\mu}}\right|$. Note that the errors on $\alpha_{T T}$ and $\alpha_{S_{\mu}}$ are correlated.

the 44 or $18 \mathrm{GHz}$ images. The effects of low $\mathrm{S} / \mathrm{N}$ on the $\alpha_{S}$ distribution can be assessed from the histogram of the spectral indices over the entire map. In Figure 12, the black curve is the Gaussian fit over the entire $\alpha_{S}$ range, which yields median value $\alpha_{S_{\mu}}=-0.78$ and standard deviation $\sigma=0.39$. Spectral indices $\alpha_{S} \leqslant \alpha_{S_{\mu}}-\sigma$ correspond to regions in the $44 \mathrm{GHz}$ image of low flux density ( $\mathrm{S} / \mathrm{N}<100)$, while $\alpha_{S} \geqslant \alpha_{S_{\mu}}+\sigma$ are associated with regions of the $18 \mathrm{GHz}$ image where $\mathrm{S} / \mathrm{N}<100$. In Figure 12, the blue curve is a better-fitting Gaussian function for $\alpha_{S_{\mu}}-\sigma \leqslant \alpha_{S} \leqslant \alpha_{S_{\mu}}+\sigma$.

The $T-T$ plots are used to measure the dominant spectral index within $0.5 \times 0.5$ squared regions, as designated in Figure 13 . The resultant $\alpha_{T T}$ values are listed in Table 4, while in Figure 14 the $T-T$ plots for each region are shown together with the histograms of the corresponding $\alpha_{S}$ distribution. In seven of the selected areas $-0.91 \leqslant \alpha_{\mathrm{TT}} \leqslant-0.72$, whereas flatter spectral indices are derived for the central region $\mathrm{C} 1\left(\alpha_{\mathrm{TT}} \sim-0.52\right)$, which includes the VLBI position of the $\mathrm{SN}$, and the adjacent northern region $\mathrm{N} 2\left(\alpha_{\mathrm{TT}} \sim-0.65\right)$. The larger differences in the temperature levels within $\mathrm{C} 1$ and N2 (see Figure 14) can be seen in the spread of the $T-T$ points, which results in the larger error bars attached to the linear fit. As shown in Figure 15, the spectral index derived for the entire remnant is $\alpha_{\mathrm{TT}_{m}}=-0.80 \pm 0.05$, where the flux calibration uncertainty is factored in the error (see Section 2).

As mentioned in Section 5.1, among the three methods used to assess the spectral index, the $T-T$ plot is the most robust, since the spectral index variations resulting from the image division and subtraction depend, to different extents, on the local intensity of the flux density at the two frequencies. However, since the $T-T$ method requires boxes larger than the angular resolution of the images, the characteristic spectral index of smaller regions, such as the features of flatter $\alpha_{t, S}$ at P.A. $\sim 30^{\circ}$, is likely influenced by surrounding steeper emission. Otherwise, when larger structures are analyzed, such as the sites of brighter emission on the eastern and western lobes, the spectral indices derived from the $T-T$ plots are likely more accurate than a local average of $\alpha_{t}$ and $\alpha_{S}$. As listed in Table 4, over the brightest sites of the remnant the discrepancy between $\alpha_{T T}$ and regional $\alpha_{S_{\mu}}$, which is the median value of the $\alpha_{S}$ distribution within the 

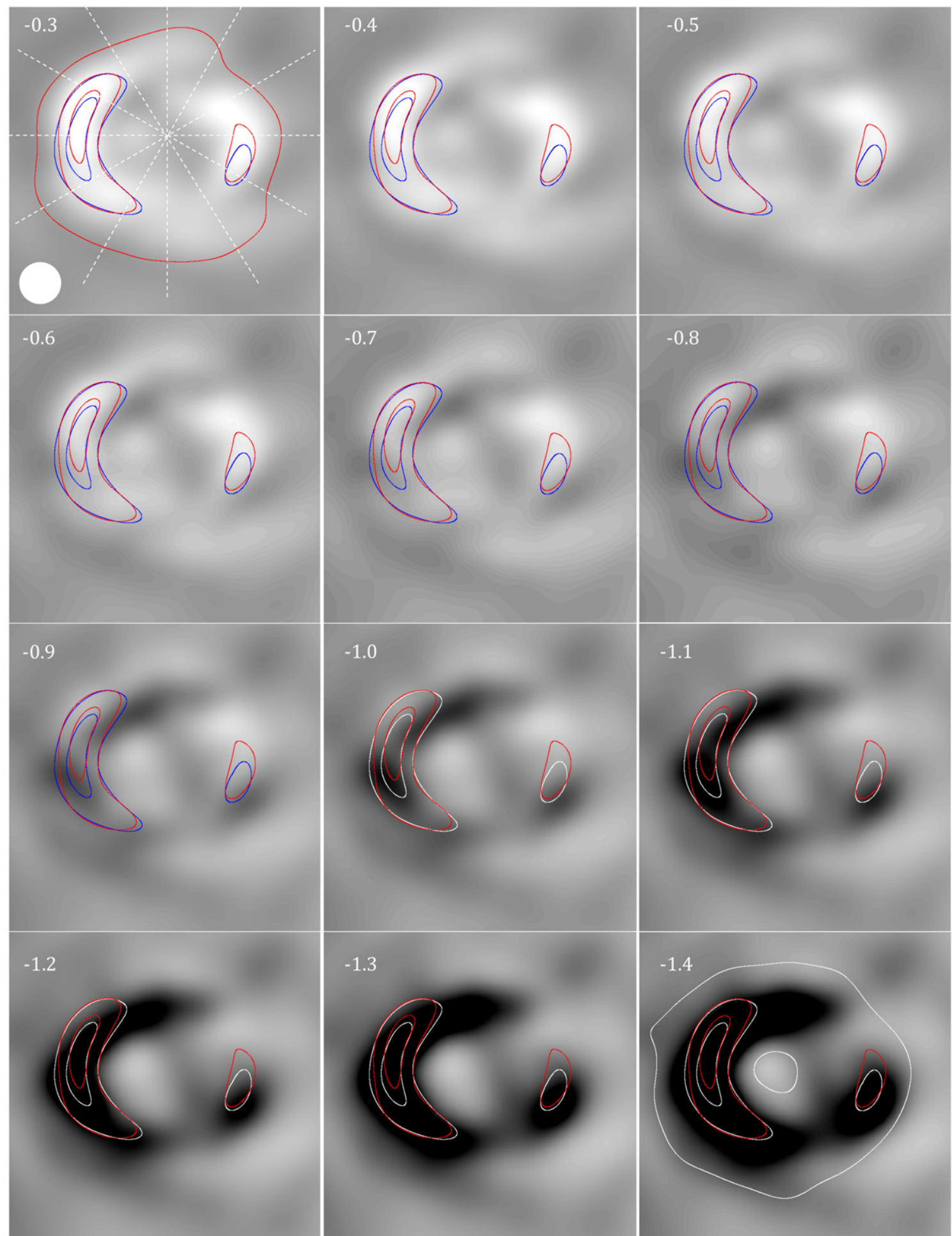

$-3.0$

$-2.0$

(mJy/beam)

0.0

1.0

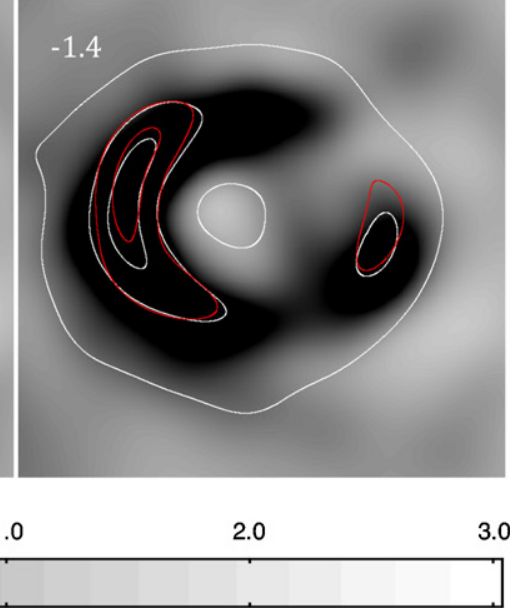

.

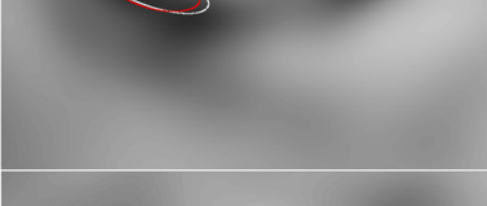

Figure 10. Tomographic spectral images of the remnant of SN 1987A, where the spectral index is defined as $S \propto v^{\alpha}$. The spectral images are derived from the $18 \mathrm{GHz}$ image from observations performed on 2011 January 26, and the $44 \mathrm{GHz}$ image from observations between in 2011 January and November. Both images are centered on the VLBI position determined by Reynolds et al. (1995), and restored with a circular 0!'4 beam (top panel, lower left corner). The gray scale shows difference images for $-1.4 \leqslant \alpha_{t} \leqslant-0.3$, in steps of 0.1 . Blue/white contours correspond to the $44 \mathrm{GHz}$ data convolved with a 0 '. 4 circular beam at levels $60 \%-90 \%$ of the peak flux density, while the related contours corresponding to the $18 \mathrm{GHz}$ image are in red. In the top left panel, axes at six position angles, centered on the SN position, are also indicated. All images are set on the same intensity range.

(A color version of this figure is available in the online journal.) 

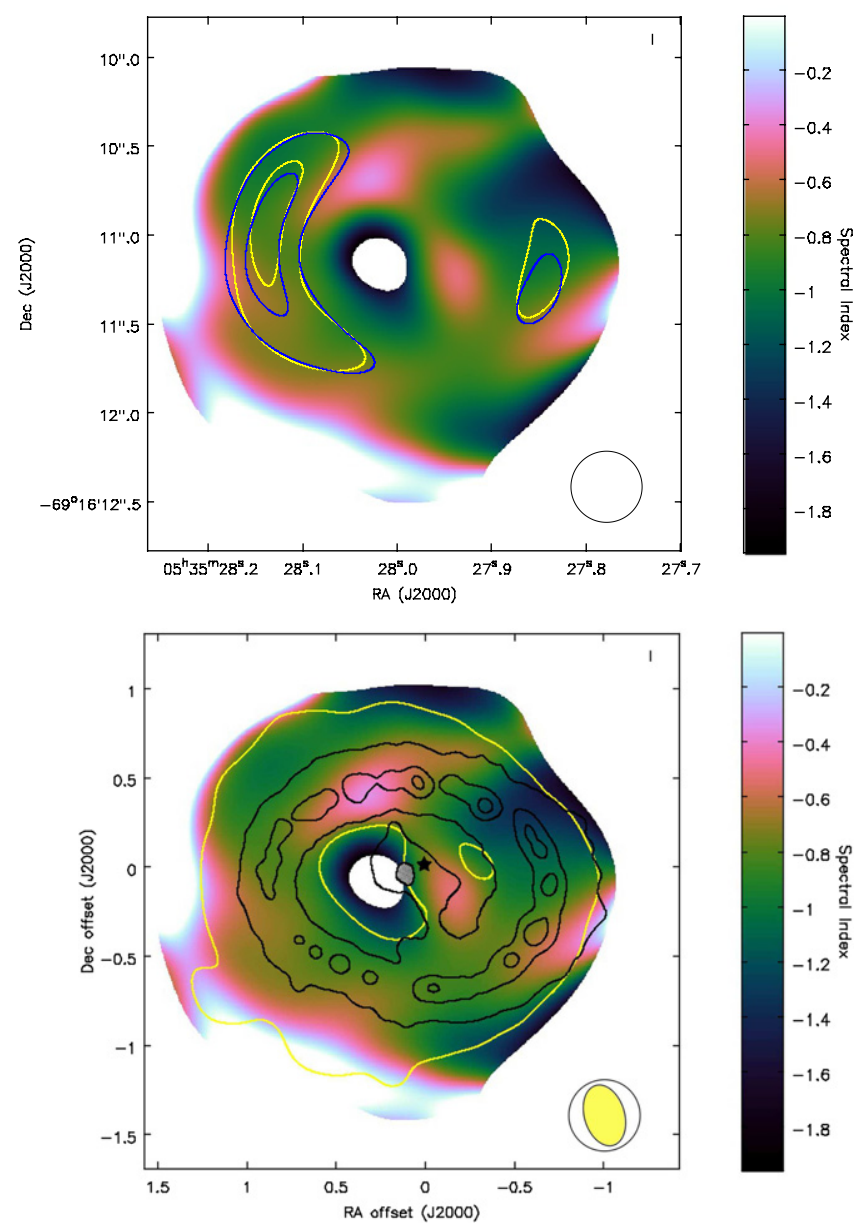

Figure 11. Top: map of the $18-44 \mathrm{GHz}$ spectral index distribution. Both the 18 and $44 \mathrm{GHz}$ Stokes $I$ images have been restored with a 0.4 circular beam, and regridded at a pixel scale of 3 mas. Image regions below the $44 \mathrm{GHz}$ rms noise level were masked. Contours of the $60 \%$ and $90 \%$ flux density levels for the $18 \mathrm{GHz}$ (yellow) and $44 \mathrm{GHz}$ (blue) images, identify the emission peaks on each lobe. Bottom: to locate the inner regions of the spectral index map with respect to the emission measured at $44 \mathrm{GHz}$, the outline of the diffraction-limited image is shown via its contour at $10 \%$ of the peak flux density (yellow). To locate the density distribution of the CSM, the spectral index map is also overlaid with the contours of the HST image derived from observations performed in 2011 (see footnote 7), specifically at the $2 \%, 7 \%, 12 \%$, and $20 \%$ emission levels (black). As indicated by Larsson et al. (2011), the innermost HST contour of the ejecta (gray fill), east of the SN position (black star), corresponds to the location of an emission drop, or "hole," in the optical image.

(A color version of this figure is available in the online journal.)

$T-T$ region, is around $19 \%$ over the eastern lobe and peaks to $\sim 31 \%$ in $\mathrm{W} 1$.

The average spectral index of the western bright regions (W1-W2) at $\sim-0.78$ matches $\alpha_{\mathrm{TT}_{m}}$. As discussed earlier, the steeper $\alpha_{S}$ values in W1 are likely affected by the higher noise levels in the adjacent northern region. Over the eastern lobe, the $T-T$ plots for regions E1 and E2 give an average spectral index of -0.86 , which is steeper than $\alpha_{\mathrm{TT}_{m}}$. The steepest value $\alpha_{\mathrm{TT}} \sim-0.91$ is associated with region E1, which covers the brightest sites of the remnant.

From the theory of diffusive shock acceleration (DSA) of energetic particles in a uniform magnetic field (see Drury 1983, and references therein), the standard compression ratio, $r$, can be derived from the radio synchrotron spectral index. The $\alpha_{T T}$ values given in Table 4 yield similar compression ratios in regions $\mathrm{W} 1, \mathrm{~W} 2$, and $\mathrm{E} 2$, specifically with $r_{\mathrm{W} 1}=2.97 \pm 0.43$,

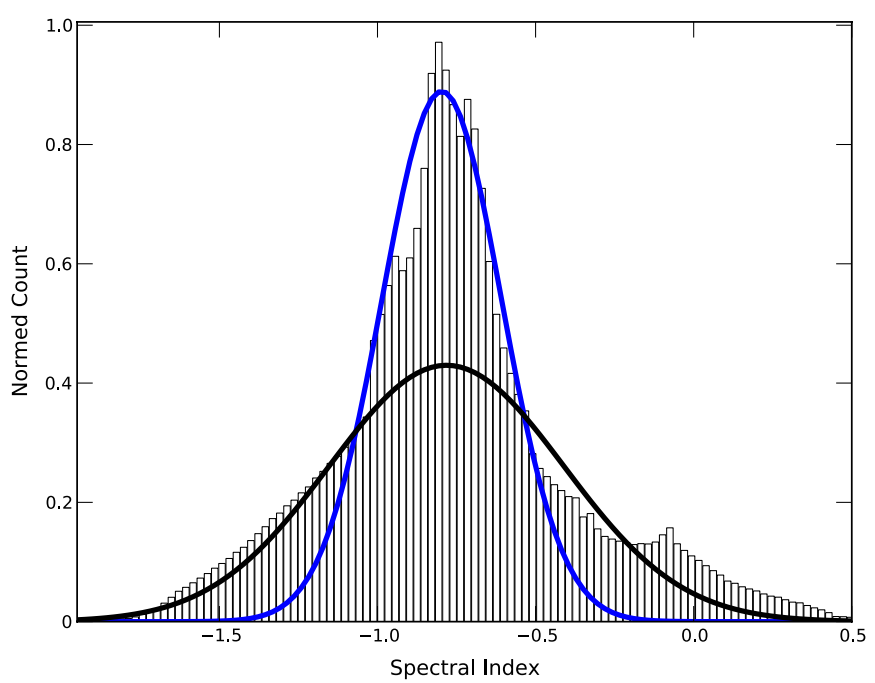

Figure 12. Histogram of the spectral index values, $\alpha_{S}$, derived from direct division of the images at 44 and $18 \mathrm{GHz}$ (see Figures 11). The vertical axis is the normalized number of occurrences. The black line is the Gaussian fit of the whole histogram, with median value $\alpha_{S_{\mu}}=-0.78$ and standard deviation $\sigma=0.39$. The blue line is the Gaussian fit for $\alpha_{S_{\mu}}-\sigma \leqslant \alpha_{S} \leqslant \alpha_{S_{\mu}}+\sigma$. Spectral indices $\alpha_{S} \leqslant \alpha_{S_{\mu}}-\sigma$ correspond to regions in the $44 \mathrm{GHz}$ image of low flux density $(\mathrm{S} / \mathrm{N}<100)$, while spectral indices $\alpha_{S} \geqslant \alpha_{S_{\mu}}+\sigma$ are associated with regions of the $18 \mathrm{GHz}$ image where $\mathrm{S} / \mathrm{N}<100$.

(A color version of this figure is available in the online journal.)

$r_{\mathrm{W} 2}=2.87 \pm 0.26$ and $r_{\mathrm{E} 2}=2.87 \pm 0.14$, while a lower compression ratio, $r_{\mathrm{E} 1}=2.65 \pm 0.13$, results in region $\mathrm{E} 1$. These results suggest a particle spectrum harder over the western regions than on the brightest eastern sites. For a particle density expressed as $n(p) \propto p^{-\gamma}$, where $p$ is the scalar momentum, in the scenario of ordinary diffusion, $n_{(\mathrm{W} 1-\mathrm{W} 2)} \propto p^{-2.56 \pm 0.26}$ is the spectrum of the western side of the SNR, while $n_{(\mathrm{E} 1)} \propto$ $p^{-2.82 \pm 0.14}$ is obtained for region E1.

The lower compression ratio in region E1 could correspond with the asymmetric expansion observed in the remnant, which appears to be driven by higher eastern velocities (see Section 4). As $r=\rho_{2} / \rho_{1}$ (Jones \& Ellison 1991), where $\rho_{2}$ and $\rho_{1}$ are the downstream and upstream gas densities, respectively, a lower compression ratio over the eastern brightest sites could be due to the higher-velocity shock drifting the cosmic rays (CR) upstream. The CR diffusion could induce gas heating upstream of the shock (Ptuskin et al. 2010) and, thus, a higher upstream pressure. If the SNR magnetic field is tangled rather than uniform (Duffy et al. 1995), the softer spectrum could be the result of local sub-diffusive particle transport, as the accelerated particles get partially trapped in the vicinity of the shock front by structures in the magnetic field (Kirk et al. 1996). In this case, the particle spectrum corresponding to region E1 would be steeper than for diffusive transport, with $n_{(\mathrm{E} 1)} \propto p^{-3.35 \pm 0.19}$, where $\gamma=\gamma_{\text {DSA }}(1+0.5 / r)$ (Kirk et al. 1996).

The softer spectrum in E1 likely implies that a lower fraction of injected particles, i.e., a higher injection efficiency, is required for the high emission observed in the eastern region. If the stronger eastbound shock is coupled with a more efficient particle acceleration, the magnetic-field on the eastern lobe might become locally amplified (Bell \& Lucek 2001; Bell 2004). In fact, according to Bell \& Lucek (2001), with efficient CR acceleration, $(\Delta B / B)^{2} \approx \mathcal{M}$, where $B$ and $\Delta B$ are the background and fluctuating magnetic fields, respectively, and $\mathcal{M}$ is the Mach number of the shock. Thus, a higher $\mathcal{M}$ would likely induce non-linear amplifications of the magnetic field generated 


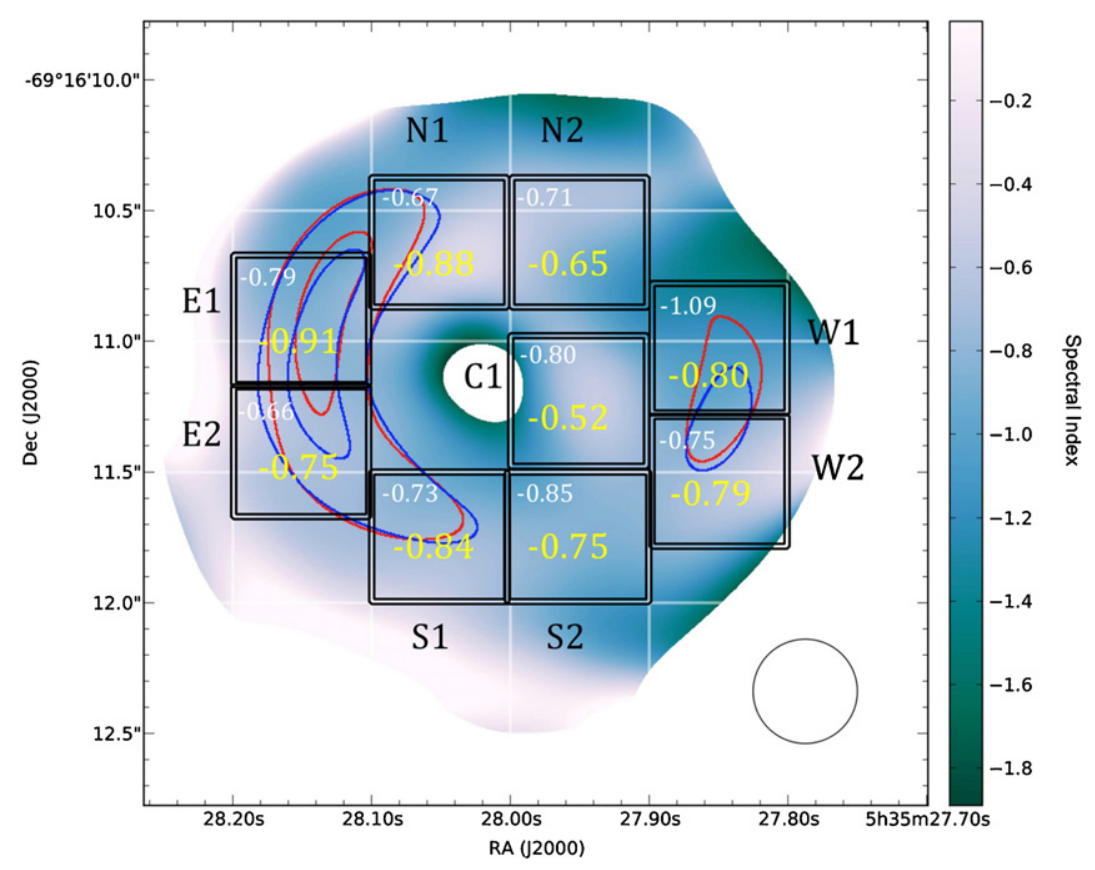

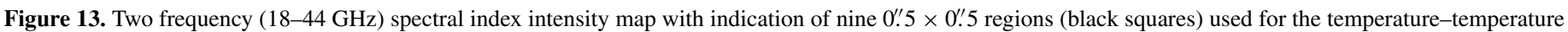

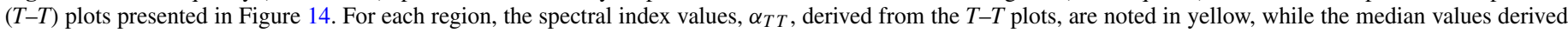

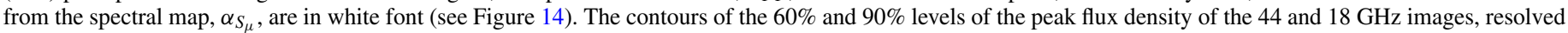
with a 0.4 circular beam, are shown in blue and red, respectively.

(A color version of this figure is available in the online journal.)

in the SNR. As the magnetic-field amplification depends on the magnetic field orientation, it can be noted that in asymmetric bipolar (or bilateral) remnants, such as SNR 1987A, regions of higher emission have been linked to either quasi-parallel or quasi-perpendicular magnetic field inclinations (Fulbright \& Reynolds 1990; Gaensler 1998; Orlando et al. 2007).

Regarding the $\alpha_{T T}$ results in the northern and southern parts of the SNR, adjacent small-scale features of both steeper and flatter spectra, lead to spectral indices flatter than $\alpha_{\mathrm{TT}_{m}}$ in the $\mathrm{S} 2$ and N2 regions, while the values derived for regions N1 and $\mathrm{S} 1$ seem still dominated by the steeper indices associated with the bright sites on the eastern lobe. We note that, overall, the spectral indices over the high-emissivity regions are steeper than predicted by first-order Fermi acceleration at a strong shock. This agrees with studies on the spectral distribution of young and rapidly evolving remnants (e.g., see discussion on Cas A in Anderson et al. 1991), where, since concurrent high-emissivity and steep spectral indices cannot be obtained from first-order acceleration models, other mechanisms, such as CR-mediated shocks and turbulent acceleration, are invoked (Anderson \& Rudnick 1993).

In both $\alpha_{t}$ and $\alpha_{S}$ distributions, gradual transitions from steeper to flatter spectral indices (i.e., $-0.8 \lesssim \alpha_{t, S} \lesssim-0.5$ ) can be seen on the edges of the remnant and around or in proximity to the brightest sites. Spectral index gradients in older and larger SNRs have been explained as due to multiple overlapping spectral index structures (DeLaney et al. 2002; Tam et al. 2002). According to hydrodynamic simulations coupled with DSA by Ellison et al. (2007) of a spherical SNR, a spectral gradient might reflect a spectral index structure over multiple concentric shells of shocked material within the forward and the reverse shocks, with a CR population varying between the concentric shells, such that the spectral index results flatter on the outer shell than on the inner ones. The scenario of sub-diffusive transport of particles at the shock front, also leads to small-scale spectral gradients in the downstream plasma, which would correspond to the transition between sub-diffusive and diffusive behavior of the particle propagation (Kirk et al. 1996).

All methods used for spectral analysis show two inner regions of flatter spectral indices, where $-0.6 \lesssim \alpha_{\mathrm{TT}} \lesssim-0.5$ and $-0.5 \lesssim \alpha_{t, S} \lesssim-0.3$. The more central feature, which overlaps with the SN site (see Figure 11), has $\alpha_{t} \approx-0.5$, $-0.6 \lesssim \alpha_{S} \leqslant-0.5$ and $\alpha_{\mathrm{TT}} \sim-0.52 \pm 0.18$. The second feature, located farther north, can be associated with $\alpha_{t} \approx-0.4$ and $-0.4 \lesssim \alpha_{S} \lesssim-0.3$, while the corresponding $\alpha_{T T}$, between regions $\mathrm{N} 1$ and $\mathrm{N} 2$, is affected by the nearby steeper spectra.

We note that a central feature of flatter spectrum has been seen in other two-frequency spectral index images of the SNR (Potter et al. 2009; Lakićević et al. 2012). In Figure 11 the spectral index image is also overlaid with the contours of the $H S T$ observations in $2011^{7}$ (in black), and with the $10 \%$ level of the peak flux density of the high-resolution image at $44 \mathrm{GHz}$ (in yellow). It can be seen that the central feature of flatter electron spectrum, not only coincides with the inner region of fainter emission that is visible in the radio at higher resolution, but also partially overlaps with the western side of the optical ejecta. On the contrary, the northern feature, which appears to connect with the central feature along P.A. $\sim 30^{\circ}$, approximately in alignment with the main direction of the optical ejecta, does not seem to have been noted before. This feature exhibits $\alpha_{t, S}$ values flatter than the central region, and, since it appears to be smaller than the $0.5 \times 0$ ' .5 boxes used for the $T-T$ plots, cannot be associated with a local $\alpha_{T T}$ value. Nevertheless, since it corresponds to a site characterized by high flux density both at 18 and $44 \mathrm{GHz}$, the related $\alpha_{t}$ and $\alpha_{S}$ values are unlikely affected by significant error.

\footnotetext{
7 STScI-2011-21, NASA, ESA, \& Challis P. (Harvard-Smithsonian Center for Astrophysics)

http://hubblesite.org/newscenter/archive/releases/2011/21/image/
} 

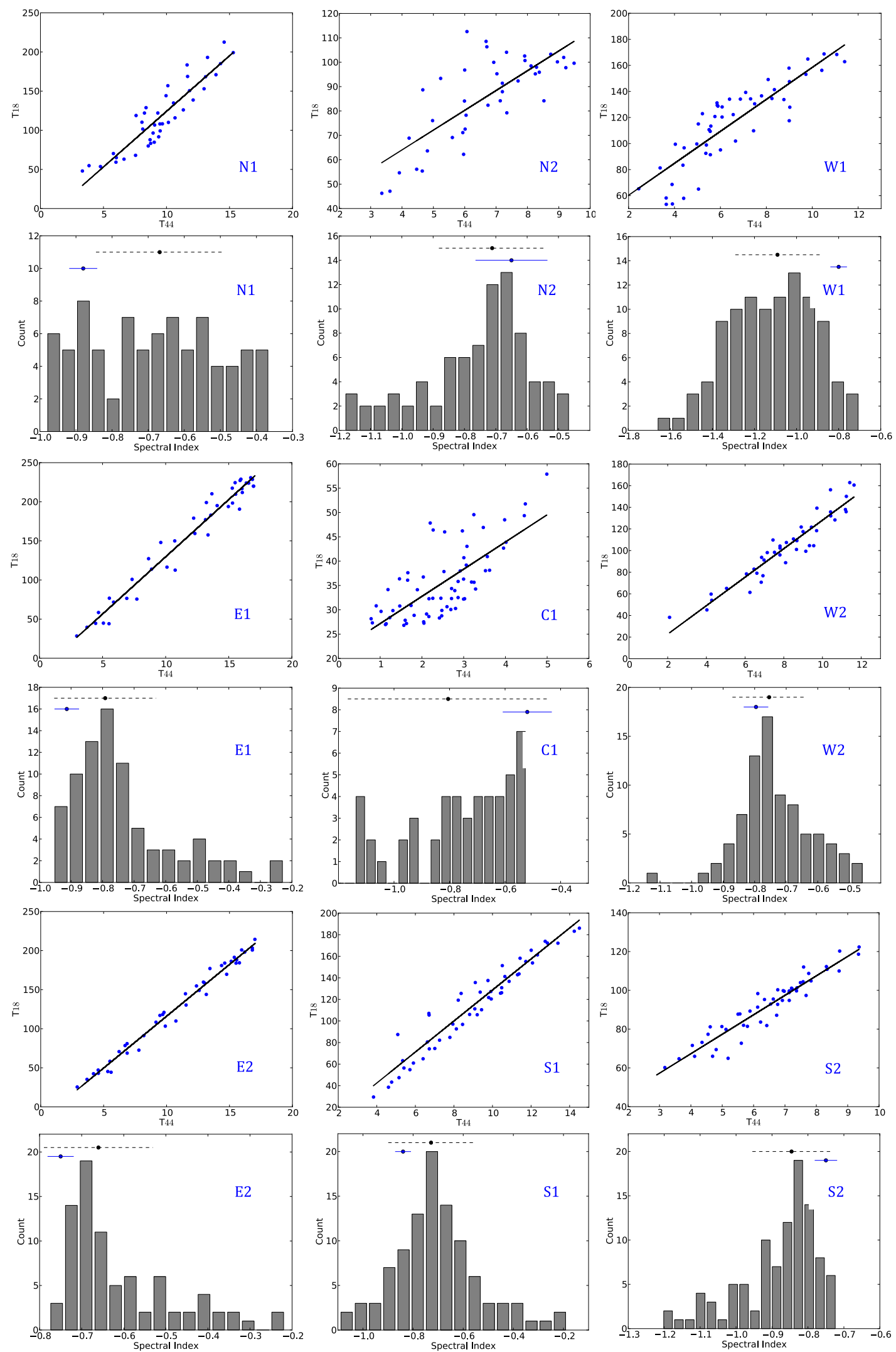

Figure 14. Two-frequency $(18-44 \mathrm{GHz})$ temperature-temperature $(T-T)$ plots for nine remnant regions, designated as in Figure 13 . For each region, the histogram of the distribution of the spectral index values, $\alpha_{S}$, is also shown, as obtained from the spectral index intensity map. In each histogram figure, the solid black circle indicates the related median spectral index and the dashed black line corresponds to the standard deviation, as derived from the Gaussian fit of the histogram. The solid blue circle indicates the spectral index derived from the linear-regression fit of each $T-T$ plot, $\alpha_{T T}$, and the blue bar indicates the $1 \sigma$ error. The values of $\alpha_{T T}$ and $\alpha_{S_{\mu}}$ associated with each region are listed in Table 4.

(A color version of this figure is available in the online journal.) 


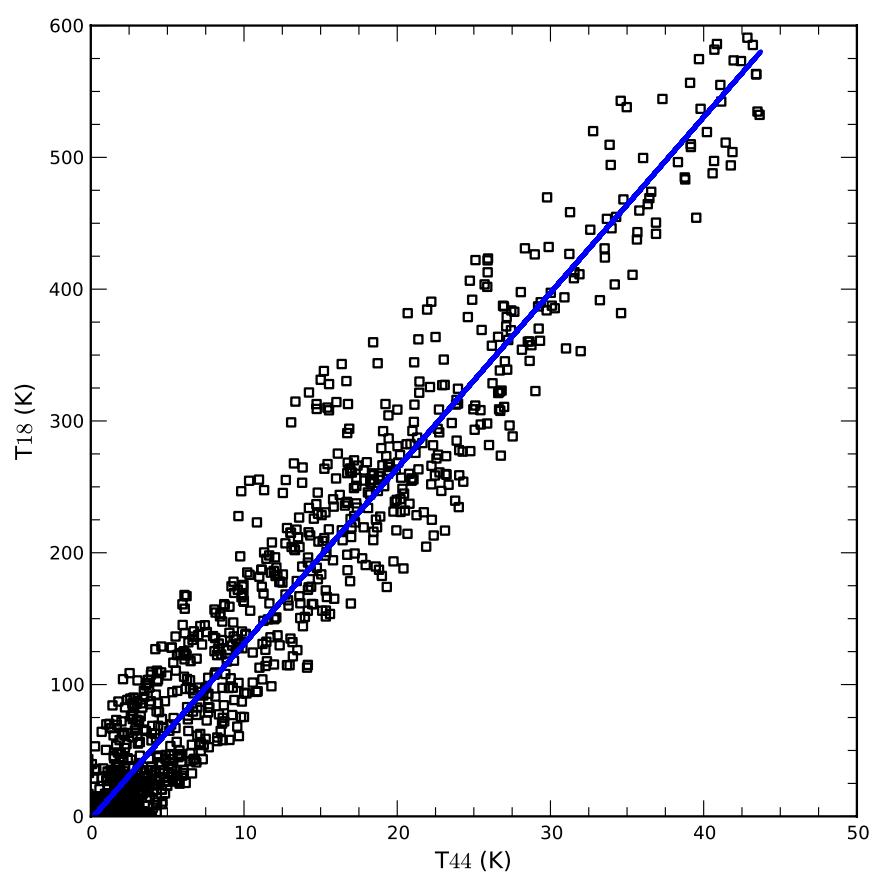

Figure 15. Temperature-temperature $(T-T)$ plot of the whole SNR at $44 \mathrm{GHz}$ and $18 \mathrm{GHz}$ (black squares). The best-fitting straight line corresponds to $\alpha=-0.80 \pm 0.01( \pm 0.05)\left(S=v^{\alpha}\right)$, where the two errors represent random and absolute flux density scale errors, respectively.

(A color version of this figure is available in the online journal.)

While pulsar wind nebulae (PWNe) in the radio band are identified by $-0.3 \lesssim \alpha \lesssim 0$ (Gaensler \& Slane 2006), the acceleration origin in the above discussed features of $-0.5 \lesssim$ $\alpha \lesssim-0.3$, could be explained in terms of injection by a pulsar. Further observations at high frequencies and monitoring of any morphology changes is required to clarify the nature of these features.

\section{EMISSION AT OTHER WAVELENGTHS}

As shown in early models of Type II SNRs (Chevalier 1982), the SN-CSM interaction in SNR 1987A has generated a main double-shock structure, which includes, from the outside inward, the forward shock and the reverse shock. Between the forward and reverse shocks, reflected shocks should be present due to the forward blast wave encountering the high-density CSM in the ER (Borkowski et al. 1997). While the structure of the shock can be probed by comparing the remnant morphology in the radio and X-ray wavelengths, the distribution of the progenitor dense material can be investigated via $\mathrm{H} \alpha$ images.

The radio emission mainly originates at the forward shock as this collides with the dense CSM associated with the ER, thus creating a discontinuity in the magnetic field where particles are accelerated (Zanardo et al. 2010). The forward shock can be identified with the sharp outer edge of the SNR shell, which propagates into the CSM of spatially varying density. A similar scenario happens at the reverse shock or inner edge of the SNR envelope which is normally dominated by X-ray emission. Between the reverse shock and the ER, the reflected shocks lead to the higher temperatures of the shocked gas and a flatter density profile. Up to day $\sim 4000$, the X-ray emission has likely been generated by the interactions with the low-density HII region located on the inside of the ER (Chevalier \& Dwarkadas 1995) whereas, in the last $\sim 5000$ days, it has primarily originated from the interaction with the dense inner ring (Park et al. 2011).

In Figure 16, the $44 \mathrm{GHz}$ image is compared to recent X-ray observations (Helder et al. 2013). As noted by $\mathrm{Ng}$ et al. (2009), the X-ray emission exhibits an east-west asymmetry less marked than what seen in the radio, while the north-south asymmetry is significant. In particular, the brightest X-ray site, which appears in the NE quadrant between P.A. $30^{\circ}$ and $60^{\circ}$, overlaps with the brighter regions on the eastern lobe of the $44 \mathrm{GHz}$ image, but peaks slightly northward of the eastern radio peak. In the western lobe, the X-ray emission peaks at P.A. $\sim 270^{\circ}$ and between P.A. $300^{\circ}$ and $330^{\circ}$. While the radio image is also reaching the western peak of brightness at P.A. $\sim 270^{\circ}$, the region between P.A. $300^{\circ}$ and $330^{\circ}$ corresponds to one of the fainter sites (see Section 3.1). The offset between the X-ray and radio peaks is demonstrated in the polar projection of the two images shown in Figure 16.

The $44 \mathrm{GHz}$ map and the HST image from recent observations are superimposed in Figure 17. It can be seen that the morphology of the radio emission matches the ring optical features. As
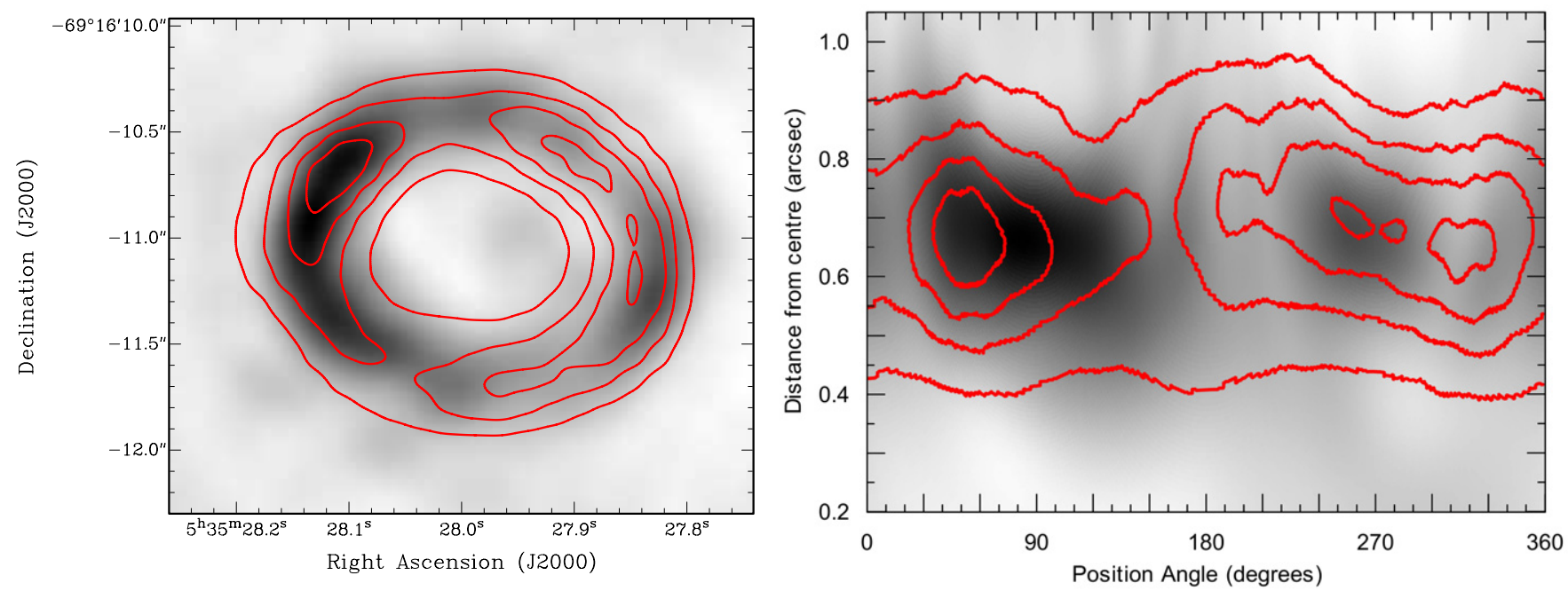

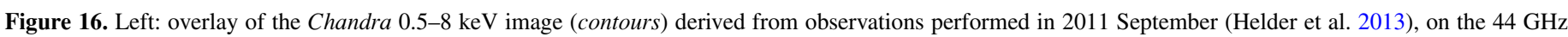

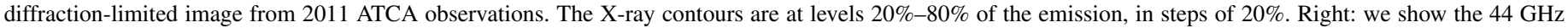

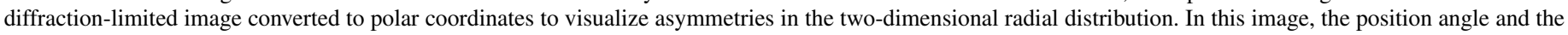
projected radial distance from the geometrical center of the remnant, are the new coordinates. The emission intensity is conserved in the conversion.

(A color version of this figure is available in the online journal.) 


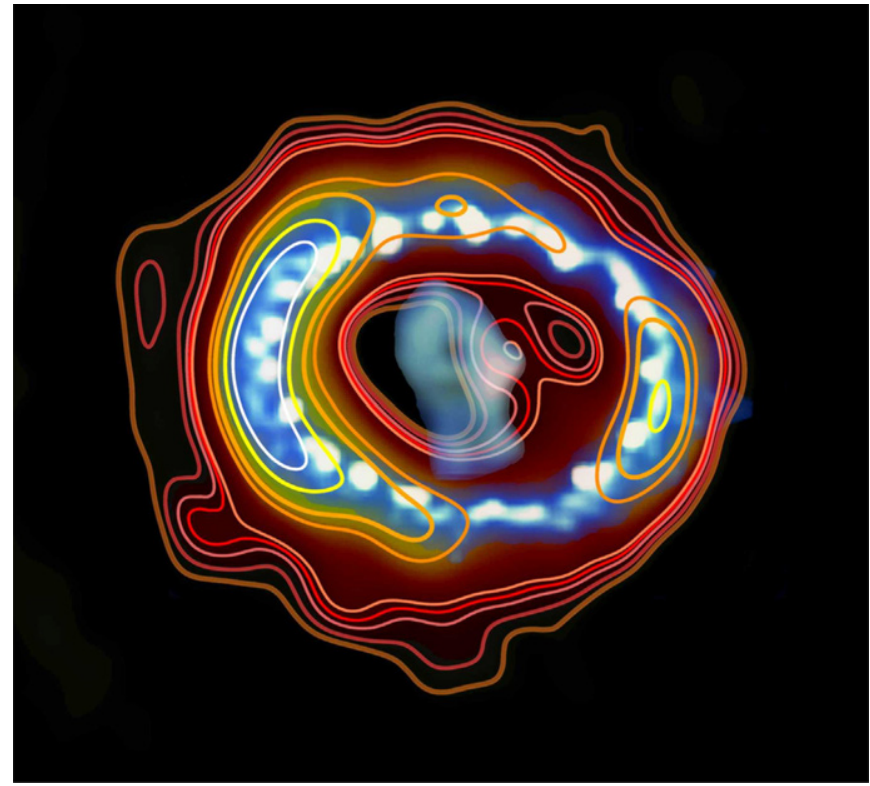

Figure 17. Overlay of the $44 \mathrm{GHz}$ image produced from ATCA observations performed in 2011 January and November (brown-yellow color scale for shades and contours) on the HST image derived from 2011 observations $^{7}$ (blue-white color scale).

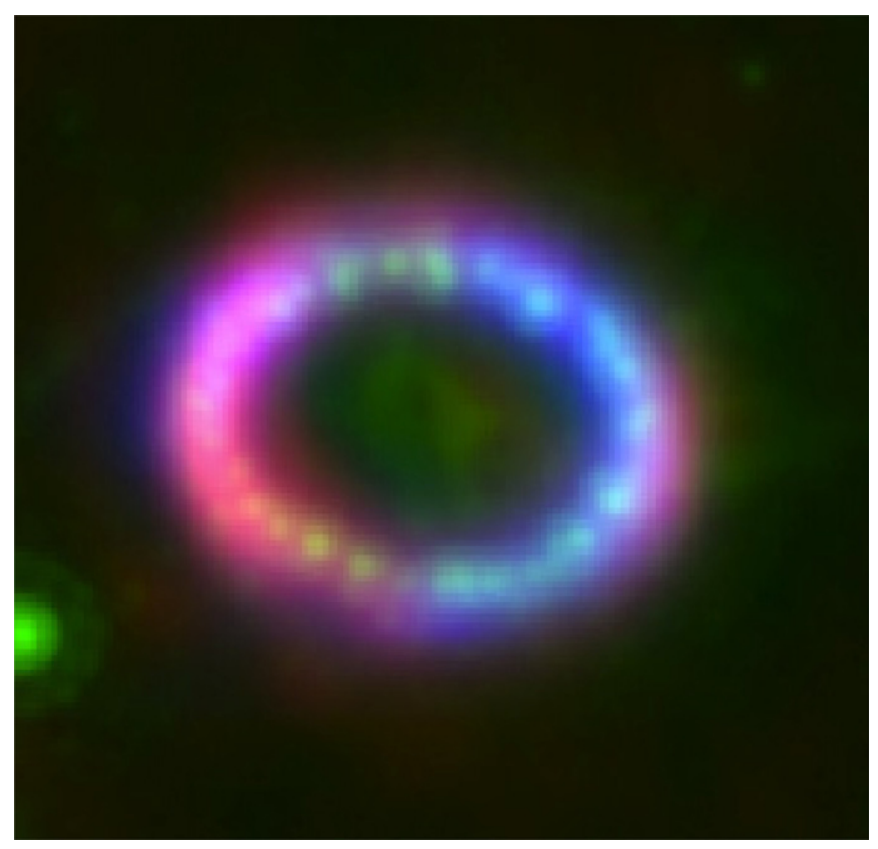

Figure 18. RGB overlay of the optical image from $2011 \mathrm{HST}$ observations 7 (green), the X-ray image from Chandra observations performed in 2011 (Helder et al. 2013; blue) and the $44 \mathrm{GHz}$ image from ATCA observations in 2011 January and November (red).

shown in Figure 11, on both the east and west sides of the optical ring, the easternmost and westernmost hot spots appear to overlap with the bright regions on the eastern and western lobes of the radio images. The fainter regions of the ring visible at $44 \mathrm{GHz}$, i.e., located in the NW and SW quadrants, seem to coincide with sites where clusters of $\mathrm{H} \alpha$ hot spots appear somewhat disjointed. This is especially pronounced on the NW quadrant, between P.A. $300^{\circ}$ and $330^{\circ}$, where the forward shock might have overtaken the ER and reached less dense CSM regions, while the inner surface of the ring is still hot enough to emit X-rays.
Regarding the remnant asymmetry, contrary to the radio images, the optical emission now appears markedly brighter on the western lobe. The difference in the asymmetry direction between the nonthermal radiation and the $\mathrm{H} \alpha$ emission is more clear in the RGB overlay shown in Figure 18. It can be noted that the eastern lobe of the ring is dominated by the radio (red) and X-ray (blue) emissions, which turn into shades of violet in the overlay, especially in the NE quadrant where the radio and $\mathrm{X}$-ray brighter sites overlap. On the western lobe, the fainter radio emission is now overshadowed by the $\mathrm{H} \alpha$ emission (green). This appears to validate the hypothesis that the remnant asymmetry is likely due to an asymmetric explosion of the progenitor rather than to an asymmetric distribution of the CSM. We may therefore expect the radio and X-ray emission to follow suit, with the east-west asymmetry gradually reversing. The possibility of an asymmetric initial explosion was suggested by Chevalier \& Soker (1989) to explain the asymmetric expansion of the remnant, whereas hydrodynamic calculations of the remnant evolution from the asymmetric explosion of a $\sim 20 M_{\odot}$ merger of binary systems, have been proposed to explain the BSG progenitor and to link the BSG-RSG wind interaction to the triple-ring nebula structure (Podsiadlowski et al. 2007; Morris \& Podsiadlowski 2007, 2009).

In Figure 17, the radio emission from the inner region of the remnant as seen at $44 \mathrm{GHz}$ can be compared to the optical emission from the ejecta. It is noted that the ejecta is blue and red-shifted along the line-of-sight (Kjær et al. 2010) and, like the emission from the ER, the optical ejecta is characterized by an east-west asymmetric morphology, whose origin is yet unclear (Larsson et al. 2011). The western side of the ejecta appears to overlap with the eastern part of the central feature visible at $44 \mathrm{GHz}$ and, as mentioned in Section 5.2, extends over a region of the SNR that can be associated with flatter spectral indices. Moreover, the "hole" in the ejecta, which is close to the SN location (see Figure 11) and has become more pronounced in recent years (Larsson et al. 2011), also overlaps with the central emission detected at $44 \mathrm{GHz}$.

\section{FREE-FREE ABSORBED COMPACT SOURCE}

Matsuura et al. (2011) have estimated that the largest dust component, with mass $0.4-0.7 M_{\odot}$, sits in the central region of the SNR. However Lakićević et al. (2012) have concluded that, for observations up to $94 \mathrm{GHz}$, the dust does not contribute to the radio emission. The central feature detected at $44 \mathrm{GHz}$ might therefore originate from a compact source or PWN located in the vicinity of the $\mathrm{SN}$ ejecta.

To determine whether non-thermal radio emission from a pulsar or a PWN located in the inner regions of the SNR would be detectable, we estimate the fraction of this emission that would be absorbed by the ionized gas likely located within the ER. Assuming the ionized mass in the ejecta, $M_{e j}$, to be distributed within a sphere of radius $R$, the free-free optical depth, $\tau_{f f}$, is proportional to the path length through the free-free absorbing spherical region along the line-of-sight (los). In convenient astronomical units, $\tau_{f f}$ can be estimated as (Rybicki \& Lightman 1979)

$$
\tau_{f f} \approx \tau_{0} T^{-3 / 2} v^{-2} \overline{g_{f f}} E M
$$

where $T$ is the temperature of the plasma in $\mathrm{K}, v$ is the frequency of the flux density in $\mathrm{GHz}, \overline{g_{f f}}=\overline{g_{f f}}(\nu, T)$ is the velocityaveraged Gaunt factor appropriate to the observing frequency, 


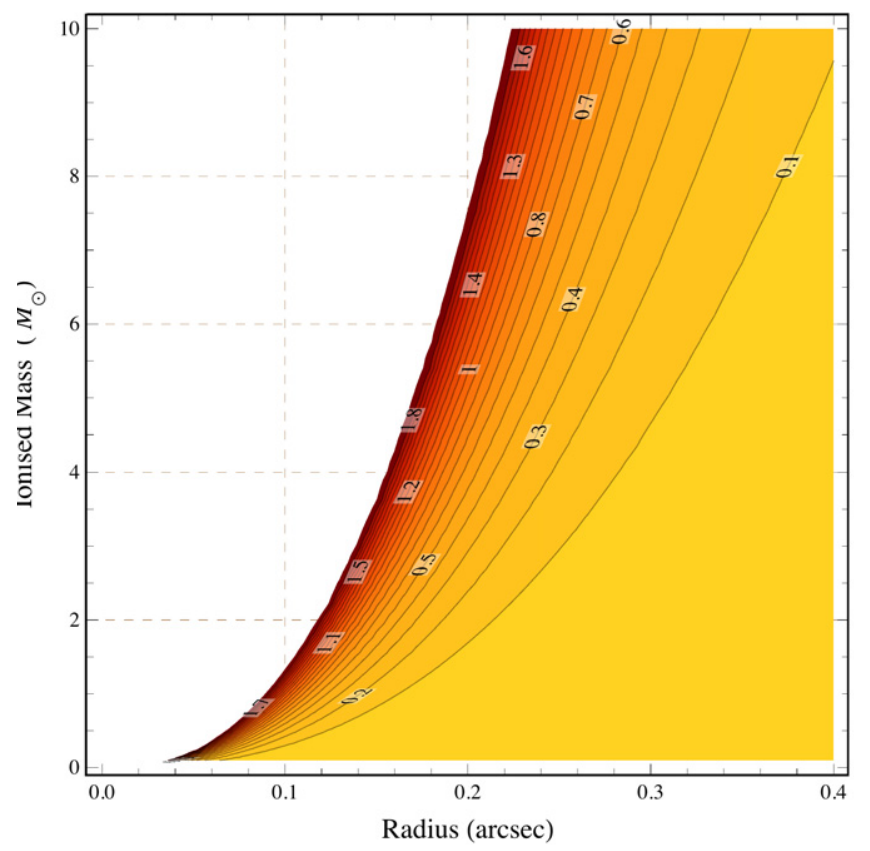

Figure 19. Variation of the optical depth, $\tau_{f f}$, of an idealized HII region in SNR 1987A, approximately located at the ejecta site. The ionized mass in the region is assumed to vary in the range $0<M_{e j} \lesssim 10 M_{\odot}$, and to be uniformly distributed within a spherical volume of size $0<R \lesssim 0^{\prime \prime} 4$, which corresponds to a path length up to $0.1 \mathrm{pc}$ along the line-of-sight. The yellow color indicates low free-free opacity; the brown color indicates high opacity.

(A color version of this figure is available in the online journal.)

and the emission measure is defined as

$$
E M \equiv \int_{\operatorname{los}} n_{e} n_{i} d l
$$

where $n_{e}$ and $n_{i}$ are the electron and ion densities, respectively, expressed in $\mathrm{cm}^{-3}$, and $l$ is the path length, expressed in pc, along the line-of-sight. As Hir regions typically have electron temperatures of order $10^{4} \mathrm{~K}$, indicated by $T_{4}$, and, in the radio regime, $\overline{g_{f f}}(v, T) \approx 5.99 T_{4}{ }^{0.15} v^{-0.1}$ (Brown 1987), the free-free opacity becomes

$$
\tau_{f f} \approx 3.28 \times 10^{-7} T_{4}^{-1.35} v^{-2.1} n_{e}^{2} R
$$

assuming $n_{e}=n_{i}$, which is approximated by $n_{e}\left(M_{e j}, R\right) \approx$ $3 M_{e j}\left(m_{p} 4 \pi R^{3}\right)^{-1}$, and $\int_{\operatorname{los}} d l \approx R$.

Figure 19 shows the variation of $\tau_{f f}$ as a function of the ionized mass for $M_{e j} \lesssim 10 M_{\odot}$ and volume size $R \leqslant 0.1 \mathrm{pc}$. The contour levels are plotted for $0 \leqslant \tau_{f f} \leqslant 2$. According to this simplified model, an ionized mass in excess of $5 M_{\odot}$ within a radius of $0^{\prime \prime} 2$ (approximately corresponding to the size of the densest part of the ejecta visible with $H S T$ ), is required to produce a free-free optical depth of unity at $44 \mathrm{GHz}$. We note that the flux density from the emitting plasma, within a $\sim 0^{\prime \prime} .2$ solid angle, given by $S_{M_{e j}}=\left(2 k_{B} T / \lambda^{2}\right) \Omega$, is negligible. In this scenario, the radiation observed through the region of ionized plasma would be $S_{\text {obs }} \approx 0.63 S_{44 c}$, where $S_{44 c}$ is the actual flux density at $44 \mathrm{GHz}$ associated with the compact source in the background. Therefore, since the flux density measured in the inner region of the $44 \mathrm{GHz}$ image is $S_{\mathrm{obs}} \sim 1.4 \mathrm{mJy}$ (see Section 3.1), the actual radio emission originating from a compact source inside the ER could be as high as $\sim 2.2 \mathrm{mJy}$. Using the spectral index derived in Figure 6, this emission would lead to $S_{94 c} \sim 1.3 \mathrm{mJy}$ at $94 \mathrm{GHz}$, which is consistent with the upper limit of $1 \mathrm{mJy}$ set by Lakićević et al. (2012) at that frequency, for any discrete radio source in the central region of the remnant.

In the case of a more realistic, steeper density profile, such as that proposed by Chevalier \& Soker (1989) with $n_{e} \propto R^{-9.6}$, the emission from a PWN would be more heavily absorbed and could be detected only if there are holes in the ejecta. As it has been noted from HST images in the $R$-band and $B$-band, the ejecta is likely to have holes (Larsson et al. 2011) (see Figures 17 and 18), thus the radiation emitted by a PWN or compact source might escape the dense ionized material and become visible at lower frequencies. On the other hand, with a more compact density distribution, part of $M_{e j}$ may have fallen back onto the neutron star that formed in the explosion, and, subsequently, the neutron star and any accreted ejecta might have already collapsed into a black hole.

\section{CONCLUSIONS}

We have presented the first image of SNR 1987A at $44 \mathrm{GHz}$, as derived from ATCA observations performed in 2011. To investigate the spectral index distribution across the remnant, this new image has been analyzed in conjunction with that derived from contemporaneous observations at $18 \mathrm{GHz}$. The emission morphology has been also compared to contemporaneous optical and X-ray observations. A summary of our findings is below.

1. Consistent with previous radio observations, the $44 \mathrm{GHz}$ image shows a marked asymmetry in the emission distribution. More specifically, the east-west asymmetry ratio is $\sim 1.5$ from the ratio of the brightness peaks in the radial profiles at P.A. $\sim 90^{\circ}$ is $\sim 1.6$ from the integrated flux densities over the eastern and western halves of the image. These values are higher than the $\sim 1.4$ ratio derived for the new $18 \mathrm{GHz}$ image and the ratio previously measured with images at lower frequencies.

2. The comparison between the new images at both 18 and $44 \mathrm{GHz}$ with corresponding observations performed in earlier epochs, specifically the 2003 observations at 17 and $19 \mathrm{GHz}$ and the 2008 observations at $36 \mathrm{GHz}$, highlights an asymmetric expansion of the remnant, with expansion velocities on the eastern lobe significantly higher than what measured on the western lobe.

3. The 18-44 GHz spectral index distribution is measured at an angular resolution of 0.4 . The spectral indices in SNR 1987A primarily range between -1.1 and -0.3 , with a mean of -0.8 . Spectral indices associated with the brightest sites over the eastern lobe are steeper than the mean value. The steeper spectrum on the eastern lobe implies compression ratios slightly lower than on the western bright sites, and could be correlated with the higher expansion rate measured on the eastern side of the remnant. Two regions of flatter spectral indices are identified, one approximately located in the center of the SNR and the other located further north. These two features lie at P.A. $\sim 30^{\circ}$.

4. There is a strong correspondence between major features of the emission at $44 \mathrm{GHz}$, and the arrangement of the hot spots shown in the $\mathrm{H} \alpha$ emission. The direction of the east-west asymmetry of the X-ray and radio emission, is opposite to that of the $\mathrm{H} \alpha$ emission. This fact supports the hypothesis that the remnant asymmetric morphology might be due to an asymmetric explosion, rather than to an asymmetric distribution of the CSM.

5. At $44 \mathrm{GHz}$, a central feature of fainter emission appears to extend over the SN site, and to overlap with the western 
side of the ejecta as seen by HST. This feature corresponds to a region of flatter spectral indices in the $18-44 \mathrm{GHz}$ spectral map, which could indicate the presence of a compact source or a PWN. The origin of this emission is unclear. However, simple free-free absorption models suggest that the radiation emitted by a compact source inside the equatorial ring may now be detectable at high frequencies, or at lower frequencies if there are holes in the ionized component of the ejecta. Future high-resolution observations, both at lower frequencies with VLBI and at higher frequencies with ATCA and the Atacama Large subMillimeter Array, will be crucial to further investigating the nature of this emission.

We thank Ray Norris for the assistance provided as duty astronomer during the site-based ATCA observations in 2011 January and Robin Wark for assistance both on site and during the remote observing session in 2011 November. We thank Robert Kirshner and Peter Challis for providing the HST images and Eveline Helder for providing the Chandra image. G.Z. also would like to thank Gerhardt Meurer for help with the HST images and Tobias Westmeier and Attila Popping for useful feedback on radio imaging techniques. Figure 11 utilizes the cube helix color scheme introduced by Green (2011). G.Z. has been partially supported by scholarships from ICRAR and the University of Western Australia. Parts of this research were conducted by CAASTRO, through project number CE110001020. The Australia Telescope Compact Array is part of the Australia Telescope, which is funded by the Commonwealth of Australia for operation as a National Facility managed by CSIRO.

\section{REFERENCES}

Anderson, M. C., \& Rudnick, L. 1993, ApJ, 408, 514

Anderson, M. C., Rudnick, L., Leppik, P., Perley, R., \& Braun, R. 1991, ApJ, 373,146

Ball, L., Crawford, D. F., Hunstead, R. W., Klamer, I., \& McIntyre, V. J. 2001, ApJ, 549, 599

Barkat, Z., \& Wheeler, J. C. 1988, ApJ, 332, 247

Bell, A. R. 2004, MNRAS, 353, 558

Bell, A. R., \& Lucek, S. G. 2001, MNRAS, 321, 433

Blondin, J. M., \& Lundqvist, P. 1993, ApJ, 405, 337

Borkowski, K. J., Blondin, J. M., \& McCray, R. 1997, ApJ, 477, 281

Briggs, D. S. 1995, AAS, 187, 112.02 http://www.aoc.nrao.edu/dissertations/ dbriggs/

Brown, R. L. 1987, in Spectroscopy of Astrophysical Plasmas, ed. A. Dalgarno \& D. Layzer (New York: Cambridge Univ. Press), 35

Chevalier, R. A. 1982, ApJ, 259, 302

Chevalier, R. A., \& Dwarkadas, V. V. 1995, ApJL, 452, L45

Chevalier, R. A., \& Soker, N. 1989, ApJ, 341, 867

Costain, C. H. 1960, MNRAS, 120, 248

Crotts, A. P. S., \& Heathcote, R. S. 2000, ApJ, 528, 426

DeLaney, T., Koralesky, B., Rudnick, L., \& Dickel, J. R. 2002, ApJ, 580, 914

Drury, L. Oc. 1983, RPPh, 46, 973

Duffy, P., Kirk, J. G., Gallant, Y. A., \& Dendy, R. O. 1995, A\&A, 302, L21

Ellison, D. C., Patnaude, D. J., Slane, P., Blasi, P., \& Gabici, S. 2007, ApJ, 661, 879
Fulbright, M. S., \& Reynolds, S. P. 1990, ApJ, 357, 591

Gaensler, B. M. 1998, ApJ, 493, 781

Gaensler, B. M., Manchester, R. N., Staveley-Smith, L., et al. 1997, ApJ, 479, 845

Gaensler, B. M., \& Slane, P. O. 2006, ARA\&A, 44, 17

Gaensler, B. M., Staveley-Smith, L., Manchester, R. N., et al. 2007, in AIP Conf. Proc. 937, Supernova 1987A: 20 Years After: Supernovae and Gamma-Ray Bursters, ed. S. Immler, K. W. Weiler, \& R. McCray (Melville, NY: AIP), 86 Green, D. A. 2011, BASI, 39, 289

Gull, S. F., \& Daniell, G. J. 1978, Natur, 272, 686

Helder, E. A., Broos, P. S., Dewey, D., et al. 2013, ApJ, 764, 11

Högbom, J. H. 1974, A\&AS, 15, 417

Jakobsen, P., Albrecht, R., Barbieri, C., et al. 1991, ApJ, 369, 63

Jones, F. C., \& Ellison, D. C. 1991, SSRv, 58, 259

Katz-Stone, D. M., \& Rudnick, L. 1997, ApJ, 488, 146

Kirk, J. G., Duffy, P., \& Gallant, Y. A. 1996, A\&A, 314, 1010

Kjær, K., Leibundgut, B., Fransson, C., Jerkstrand, A., \& Spyromilio, J. 2010, A\&A, 517, A51

Lakićević, M., Zanardo, G., van Loon, J. Th., et al. 2012, A\&A, 541, L2

Larsson, J., Fransson, C., Östlin, G., et al. 2011, Natur, 474, L484

Leahy, D. A., \& Roger, R. S. 1991, AJ, 101, 1033

Manchester, R. N., Gaensler, B. M., Staveley-Smith, L., Kesteven, M. J., \& Tzioumis, A. K. 2005, ApJL, 628, L131

Manchester, R. N., Gaensler, B. M., Wheaton, V. C., et al. 2002, PASA, 19, 207

Martin, C. L., \& Arnett, D. 1995, ApJ, 447, 378

Matsuura, M., Dwek, E., Meixner, M., et al. 2011, Sci, 333, 1258

Middelberg, E., Sault, R. J., \& Kesteven, M. J. 2006, PASA, 23, 147

Morris, T., \& Podsiadlowski, P. 2007, Sci, 315, 1103

Morris, T., \& Podsiadlowski, P. 2009, MNRAS, 399, 515

Ng, C.-Y., Gaensler, B. M., Murray, S. S., et al. 2009, ApJL, 706, L100

Ng, C.-Y., Gaensler, B. M., Staveley-Smith, L., et al. 2008, ApJ, 684, 481

Ng, C.-Y., Potter, T. M., Staveley-Smith, L., et al. 2011, ApJL, 728, L15

Orlando, S., Bocchino, F., Reale, F., Peres, G., \& Petruk, O. 2007, A\&A, 470, 927

Park, S., Zhekov, S. A., Burrows, D. N., et al. 2011, ApJL, 733, L35

Perley, R. A., \& Butler, B. J. 2013, ApJS, 204, 19

Plait, P. C., Lundqvist, P., Chevalier, R. A., \& Kirshner, R. P. 1995, ApJ, 439, 730

Podsiadlowski, Ph., Morris, T. S., \& Ivanova, N. 2007, in AIP Conf. Proc. 937, Supernova 1987A: 20 Years After: Supernovae and Gamma-Ray Bursters, ed. S. Immler, K. Weiler, \& R. McCray (Melville, NY: AIP), 125

Potter, T. M., Staveley-Smith, L., Ng, C.-Y., et al. 2009, ApJ, 705, 261

Ptuskin, V., Zirakashvili, V., \& Seo, E.-S. 2010, ApJ, 718, 31

Reynolds, J. E., Jauncey, D. L., Staveley-Smith, L., et al. 1995, A\&A, 304, 116

Rybicki, G. B., \& Lightman, A. P. 1979, Radiative Processes in Astrophysics (New York: Wiley), 162

Sault, R. J. 2003, AT Memo 39.3/124, http://www.atnf.csiro.au/observers/ memos/AT39.3_124.pdf

Staveley-Smith, L., Briggs, D. S., Rowe, A. C. H., et al. 1993, Natur, 366, 136

Staveley-Smith, L., Gaensler, B. M., Manchester, R. N., et al. 2007, in AIP Conf. Proc. 937, Supernova 1987A: 20 Years After: Supernovae and Gamma-Ray Bursters, ed. S. Immler, K. W. Weiler, \& R. McCray (Melville, NY: AIP), 96

Staveley-Smith, L., Manchester, R. N., Kesteven, M. J., et al. 1992, Natur, 355 , 147

Tam, C., Roberts, M. S. E., \& Kaspi, V. M. 2002, ApJ, 572, 202

Tingay, S., Phillips, C., Amy, S., et al. 2009, in Proc. $8^{\text {th }}$ International e-VLBI Workshop, Proc. Sci., 100, http://pos.sissa.it/cgi-bin/reader

Turtle, A. J., Campbell-Wilson, D., Manchester, R. N., Staveley-Smith, L., \& Kesteven, M. J. 1990, IAUC, 5086, 2

Turtle, A. J., Pugh, J. F., Kenderdine, S., \& Pauliny-Toth, I. I. K. 1962, MNRAS, 124, 297

Weiler, K. M., Panagia, N., Montes, M. J., \& Sramek, R. A. 2002, ARA\&A, 40, 387

Wilson, W. E., Ferris, R. H., Axtens, P., et al. 2011, MNRAS, 416, 832

Zanardo, G., Staveley-Smith, L., Ball, L., et al. 2010, ApJ, 710, 1515

Zhang, X., Zheng, Y., Landecker, T. L., \& Higgs, L. A. 1997, A\&A, 324, 641 